\title{
Anoxic development of sapropel S1 in the Nile Fan inferred from redox sensitive proxies, Fe speciation, Fe and Mo isotopes
}

\author{
Alan Matthews ${ }^{\mathrm{a}, *}$, Irit Azrieli-Tal ${ }^{\mathrm{a}}$, Ayelet Benkovitz ${ }^{\mathrm{a}}$, Miryam Bar-Matthews ${ }^{\mathrm{b}}$, Derek Vance $^{\mathrm{c}}$, \\ Simon W. Poulton ${ }^{\mathrm{d}}$, Nadya Teutsch ${ }^{\mathrm{b}}$, Ahuva Almogi-Labin ${ }^{\mathrm{b}}$, Corey Archer ${ }^{\mathrm{c}}$ \\ ${ }^{a}$ Institute of Earth Sciences, Hebrew University of Jerusalem, Edmond Y. Safra Givat Ram Campus, 9190401 Jerusalem, Israel \\ b Geological Survey of Israel, 30 Malkhei Israel Street, Jerusalem 9550161, Israel \\ ${ }^{\mathrm{c}}$ Institute for Geochemistry and Petrology, Department of Earth Sciences, ETH Zurich, NW D81.4, Clausiusstrasse 25, 8092 Zurich, Switzerland \\ d School of Earth and Environment, University of Leeds, Leeds LS2 9JT, United Kingdom
}

\section{A R T I C L E I N F O}

\section{Keywords:}

Nile Fan sapropel S1

Fe isotope fractionation

Fe speciation

Mo isotope fractionation

Anoxic non-sulphidic waters

Porewater pyrite diagenesis

\begin{abstract}
A B S T R A C T
Redox conditions and the mechanisms of redox development are a critical aspect of Eastern Mediterranean sapropels, whose formation in oxygen-depleted waters is closely related to water column stratification at times of global sea level rise and insolation maxima. Sapropels in the Nile Fan formed at relatively shallow water depths under the influence of the monsoon-driven freshwater output from the River Nile. This work evaluates the redox evolution of Holocene sapropel S1 in VALPAMED cruise core MD9509, recovered at 880 mbsl in the NE Nile Fan, using a combination of geochemical element proxies, Fe speciation, Fe and Mo isotopes studies. The productivity and redox proxies (Ba/Al, Mo/Al, U/Al, V/Al, Sb/Al) show well-defined enrichments in the sapropel, but with a marked minimum at ca $8.2 \mathrm{ka}$ indicative of reventilation corresponding to a well known global cooling event. Peak productivity and reducing signals occur close to the initiation of sapropel formation. The proxy signals in sapropel 9509 are stronger and of longer duration than those of a second sapropel S1, recovered at the same depth, but $380 \mathrm{~km}$ to the north (MD9501), supporting the notion (suggested in previous studies) of more reduced conditions in the Nile Fan.

The $\mathrm{Mo}_{\mathrm{EF}}$ vs. $\mathrm{U}_{\mathrm{EF}}$ enrichment factor variations in core 9509 infer a transition from open marine suboxic conditions in the enclosing non-sapropel sediments to anoxic non-sulphidic water column conditions in the sapropel. Correspondingly, the highly reactive $\mathrm{Fe}$ pool $\left(\mathrm{Fe}_{\mathrm{HR}}\right)$ measured in $\mathrm{Fe}$ speciation studies is dominated by $\mathrm{Fe}$ (oxyhydr) oxide minerals in the background sediments, whereas pyrite $\left(\mathrm{Fe}_{\mathrm{py}}\right)$ becomes the dominant component of the $\mathrm{Fe}_{\mathrm{HR}}$ pool in the sapropel. Maximum $\mathrm{Fe}_{\mathrm{py}}$ values in the sapropel coincide with peak productivity and reducing conditions, implying a clear link between trace element uptake, diagenetic bacterial sulphate reduction in anoxic porewater and Fe mobilization in the sapropel. Iron isotope compositions $\left(\delta^{56} \mathrm{Fe}\right)$ in the sapropel do not show any departure from primary (marine and detrital) source sediment values, and the absence of an $\mathrm{Fe} / \mathrm{Al}$ vs. $\delta^{56} \mathrm{Fe}$ trend strongly argues against an Fe shuttle. Molybdenum isotopes, however, show marked non-conservative fractionation patterns. Background sediment $\delta^{98 / 95}$ Mo values $(0.2$ to $0.7 \%$ ) are compatible with fractionation upon absorptive uptake by Fe (oxyhydr)oxides and pyrite. In contrast, minimum $\delta^{98 / 95} \mathrm{Mo}$ values exhibited at peak sapropel (reducing and pyrite producing) conditions are most closely modeled by Mo isotope fractionation during kinetically controlled conversion of aqueous molybdate to thiomolybdate species. The conservative Fe isotope behavior/Mo isotope fractionation minima in the sapropel may be a characteristic of organic-rich sediment diagenesis below an anoxic non-sulphidic water body, without the operation of a benthic Fe shuttle.
\end{abstract}

\section{Introduction}

Eastern Mediterranean Sea sapropels are dark, organic-rich sediment layers, formed during marine anoxic events that coincided with periods of orbitally induced insolation maxima (Rossignol-Strick et al.,
1982; Rossignol-Strick, 1985; Rohling, 1994). Density stratification of the Eastern Mediterranean Sea and cessation of thermohaline deepwater mass circulation was the major physical force driving the creation of oxygen-depleted bottom water conditions (Rossignol-Strick et al., 1982; Rohling, 1994; Emeis et al., 2000; Rohling et al., 2015). Major

\footnotetext{
* Corresponding author

E-mail address: alan.matthews@mail.huji.ac.il (A. Matthews).
} 
factors leading to this water stratification were enhanced influx of freshwater from the Nile River and presently-dry North African rivers during periods of African monsoonal maxima, increased rainfall from Atlantic westerlies in the Mediterranean region, and post-glacial sea level rise (Rossignol-Strick, 1985; Rohling, 1994; Thomson et al., 1999; Kallel et al., 2000; Emeis et al., 1998, 2000, 2003; Scrivner et al., 2004; Bar-Matthews et al., 2000, 2003; de Lange et al., 2008; Osborne et al., 2008; Bar-Matthews, 2014; Grant et al., 2016). Grant et al. (2016) inferred that sea level rise during deglaciation was a major influence on the development of the youngest Holocene sapropel S1, dated at approximately 10-6 ka, whereas monsoon forcing dominated during the earlier late Pleistocene sapropels, S3, S4, and S5. A recent oceanicbiogeochemical model (Grimm et al., 2015) also indicates that a preceding period of deep-water stagnation may have been a prerequisite for bottom water anoxia development during S1.

Formation and preservation of sapropel sediments is closely contingent on the redox state of the oxygen-depleted bottom waters from which they are deposited and porewater conditions. Regional-scale anoxic bottom water conditions were inferred for Holocene sapropel S1 at water depths $>1800 \mathrm{~m}$ below sea level (mbsl) in deeper basins of the Eastern Mediterranean Sea (de Lange et al., 2008). In contrast, suboxic bottom water conditions were identified in $\mathrm{S} 1$ sediment from core MD04-2722 recovered at 1780 mbsl water depth from the continental shelf south of Cyprus (Tachikawa et al., 2015; Fig. 1). Sapropels in the Nile Fan were deposited at even shallower water depths ( $\sim 500$ to 1000 mbsl), but nevertheless show the absence of benthic foraminifera, together with evidence for enhanced Nile suspended sediment flux, pollen and spore transport (Almogi-Labin et al., 2009; Box et al., 2011; Langgut et al., 2011; van Helmond et al., 2015; Hennekam et al., 2015). Hennekam et al. (2014) inferred redox state fluctuations in overlying waters from authigenic $\mathrm{V}$ enrichments in sapropel S1 from core PC009BC within the Nile Fan (Fig. 1). However, detailed evaluation of the redox conditions during S1 formation in the Nile Fan, including those in the preceding and postdating sediments, has not yet been made. This study constrains these redox conditions through the application of redox sensitive chemical proxies, Fe isotopes, Fe speciation and Mo isotopes, to sapropel S1 of the Nile Fan.

With few exceptions (e.g., Staubwasser et al., 2013), Fe isotope fractionation during non-quantitative reduction of Fe (oxyhydr)oxides produces isotopically light Fe(II) aq (Beard et al., 2003a; Crosby et al., 2007; Johnson et al., 2005; Severmann et al., 2006; reviewed in Dauphas et al., 2017). The effects of isotopically light Fe(II) aq are most strongly seen in euxinic basins where reaction of $\mathrm{Fe}(\mathrm{II})_{\mathrm{aq}}$ with dissolved $\mathrm{H}_{2} \mathrm{~S}$ preserves the light isotopic signature in authigenic pyrite. Incorporation of pyrite, formed in the water column, into sediments leads to increased $\mathrm{Fe} / \mathrm{Al}$ and inverse trends of $\delta^{56} \mathrm{Fe}$ with $\mathrm{Fe} / \mathrm{Al}$ and $\mathrm{S}$ wt $\%$ (the 'benthic Fe shuttle': Severmann et al., 2008; Lyons et al., 2009; Homoky et al., 2009; Fehr et al., 2010; Azrieli-Tal et al., 2014). Experimental studies on abiotic Fe isotope fractionation in the system Fe

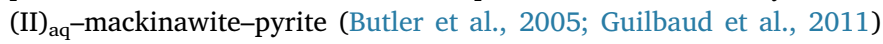
also show that isotopically light pyrite can form by kinetic isotopic fractionation pathways during sulphidization of $\mathrm{Fe}(\mathrm{II})_{\mathrm{aq}}$ and $\mathrm{FeS}$.

Redox conditions exert complex controls on Mo isotope fractionation in marine sediments (for a recent review, see Kendall et al., 2017). Whereas Mo adsorption to manganese oxides in oxic water column conditions yields light $\delta^{98 / 95}$ Mo isotopic values of $\sim-0.7 \%$ that reflect a large $\sim 3 \%$ fractionation from seawater molybdate $\left(\delta^{98}\right.$ ) ${ }^{95} \mathrm{Mo} \approx+2.3 \%$ ) (Barling et al., 2001; Siebert et al., 2003, 2006; Barling and Anbar, 2004; Wasylenki et al., 2008), sediments formed in euxinic water column conditions frequently precipitate with $\delta^{98 / 95} \mathrm{Mo}$ close to that of seawater (Arnold et al., 2004; Neubert et al., 2008). $\mathrm{H}_{2} \mathrm{~S}$ concentrations $>11 \mu \mathrm{mol}$ (Action Point of Switch (APS) of Erickson and Helz, 2000) stabilize tetrathiomolybdate $\mathrm{MoS}_{4}{ }^{2-}$ relative to other oxythiomolybdate species (Helz et al., 1996; Erickson and Helz, 2000), and are considered to bring about near complete scavenging of Mo.

When $\left[\mathrm{H}_{2} \mathrm{~S}\right]<11 \mu \mathrm{mol}$, however, dissolved Mo uptake is incomplete and Mo isotopic variation should occur due to large Mo isotope fractionations between molybdate and thiomolybdate species (Tossell, 2005; Kerl et al., 2017). The connection with dissolved sulphide was demonstrated by Neubert et al. (2008), who showed that $\delta^{98}$ ${ }^{95}$ Mo variations of -0.6 to $2.4 \%$ in Black Sea sediments from water depths $<400$ mbsl correlate with $\left[\mathrm{H}_{2} \mathrm{~S}\right]$ in adjacent waters. Azrieli-Tal et al. (2014) found light Mo isotope values $(-0.9$ to $-0.5 \%$ ) in the lower part of sapropel S1 at ODP site 967D that coincided with the lowest $\mathrm{Fe}$ isotope values and maxima in redox sensitive trace elements (RSTE). The light $\delta^{98 / 95}$ Mo values were attributed to a large negative isotopic fractionation between oceanic molybdate and tri- and tetrathiomolybdate species in low $\left[\mathrm{H}_{2} \mathrm{~S}\right]$ euxinic bottom water. Scheiderich et al. (2010) also interpreted light $\delta^{98 / 95}$ Mo and multiple S isotope values in early Pleistocene sapropels to reflect non-quantitative Mo removal from a sulphidic water column. On the other hand, Reitz et al. (2007) found an atypical light $\delta^{98 / 95}$ Mo signal ( -0.25 to $-1.1 \%$ ) in non-sulphidic sub-oxic sapropel S1 sediments, which they attributed to diagenetic overprinting of an originally oxic Mo signal. Thus,

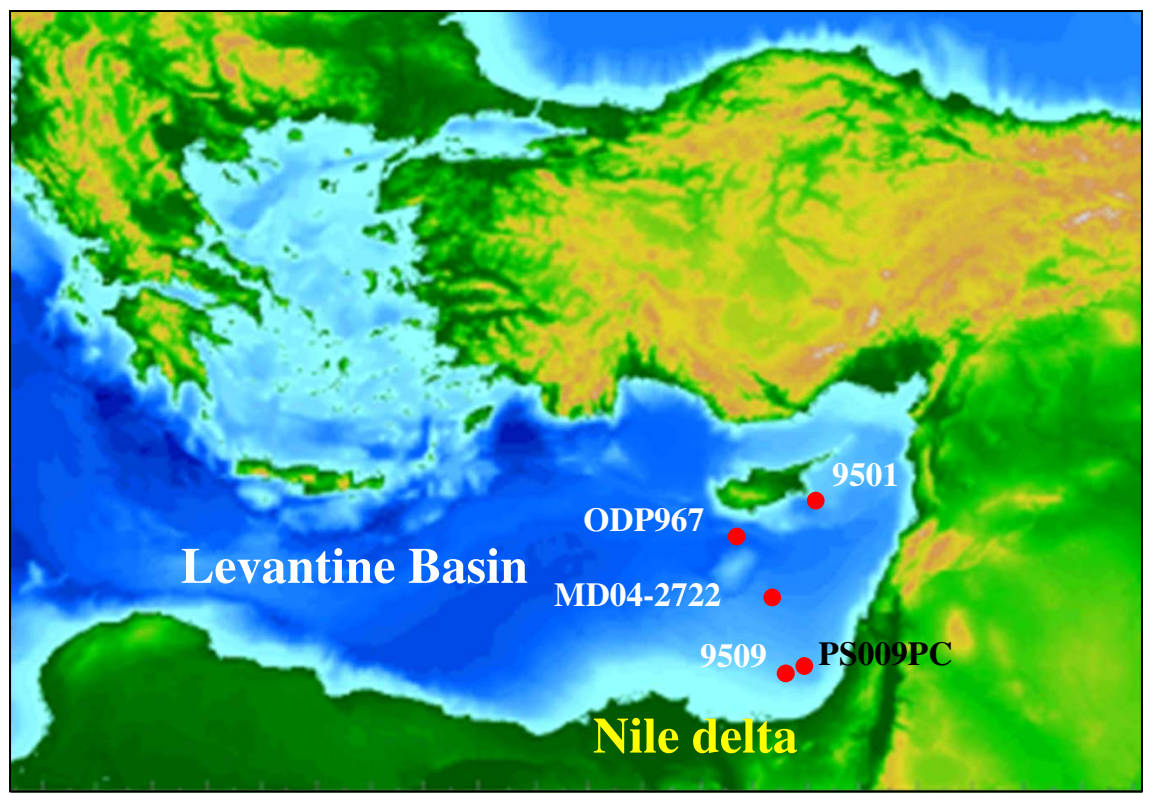

Fig. 1. Map of the Eastern Mediterranean Sea showing the location of core sites mentioned in this work: cores 9509 and 9501 (this study), ODP core 967D (Azrieli-Tal et al., 2014), MD042722 (Tachikawa et al., 2015) and PC009BC (Hennekam et al., 2015). 
depending on redox conditions, both oxic uptake by Mn oxides and the anoxic generation of thiomolybdate species can lead to isotopically light Mo in sapropels.

Although well-defined $\mathrm{Fe}$ and Mo isotope signals occur in euxinic bottom waters, such conditions may not be relevant for the shallow to intermediate depth sapropel S1 from the Nile Fan, where the primary deposition mechanism may be dominated by metal uptake from porewaters. Sediments accumulated in sub-oxic to anoxic non-sulphidic bottom waters show a wide range of $\delta^{98 / 95}$ Mo values, varying from - 0.6 to 1.6\% (Barling and Anbar, 2004; Siebert et al., 2003, 2006). Mo isotope compositions $(\sim 1.6 \%)$ of typical anoxic continental margin marine sediments (where sulphide may accumulate in porewaters) indicate a fractionation of $-0.7 \%$ from seawater Mo (Poulson et al., 2006; Poulson-Brucker et al., 2009), which matches that $\sim-0.9 \%$ determined in Mo-sulphide co-precipitation experiments (PoulsonBrucker et al., 2012). Processes such as adsorption of isotopically light Mo to Fe (oxyhydr)oxides become significant in non-sulphidic waters (Goldberg et al., 2009; Goldberg et al., 2012). Complex Fe isotope signatures reflecting both dissimilatory microbial Fe(III) reduction and bacterial sulphate reduction are recorded during modern marine sediment diagenesis (Severmann et al., 2006; Staubwasser et al., 2006; Johnson et al., 2008).

Resolving the various mechanistic alternatives for Fe and Mo isotope fractionations in marine sediments finds a powerful complement in Fe speciation studies of the relative fractions of pyrite and non-sulphidized $\mathrm{Fe}$ minerals such as $\mathrm{Fe}$ (oxyhydr)oxides and $\mathrm{Fe}$ carbonates (Poulton and Canfield, 2005, 2011). Fe speciation is a widely used geochemical tool that allows oxic and anoxic water column conditions to be distinguished, and euxinic and anoxic non-sulphidic (ferruginous) conditions can be identified (e.g., Poulton et al., 2010; Clarkson et al., 2016). Such Fe speciation studies can also indicate the presence or absence of porewater sulphide (Poulton and Canfield, 2011).

The present study is made on sapropel $\mathrm{S} 1$ and the immediate preand post-sapropel sediment from VALPAMED core MD 9509, recovered from the southern Levantine basin at the northeastern margin of the sediment-water plume of the Nile River at a water depth of $884 \mathrm{mbsl}$ (Fig. 1). Data from core MD 9501, taken from the northern Levantine basin southeast of Cyprus at a water depth of $980 \mathrm{mbsl}$, is also presented as a measure of intermediate depth sapropel S1 conditions distal from the Nile plume. These water depths correspond to the modeled intermediate zone (500-1800 mbsl) of low oxygen concentrations (Grimm et al., 2015), but lie well above the anoxic depth interval at $>1800$ mbsl. The study addresses two main issues: 1) Identification of redox conditions in the Nile Fan during the sapropel S1 event, including evidence for amplification of the sapropel redox signal in the Nile Fan relative to the wider Levantine basin at similar depths, and 2) Investigation of whether water column or diagenetic processes influence geochemical and isotope ( $\mathrm{Fe}$ and $\mathrm{Mo}$ ) signals in the sediment. Following the methodology established in other sapropel studies, we augment the metal isotopic and Fe speciation data with environmental data gained from paleoproductivity and redox geochemical proxies (Thomson et al., 1999; Calvert and Fontugne, 2001; Warning and Brumsack, 2000; Brumsack, 2006; Algeo and Tribovillard, 2009; Gallego-Torres et al., 2010).

\section{Methods}

\subsection{Core settings}

MD9509 (32.01N, 34.16E) and MD9501 (34.32N, 33.59E) are long, continuous and undisturbed cores. They were recovered from the southern Levantine basin by $\mathrm{R} / \mathrm{V}$ Marion Dufresne during the VALPAMED cruise at depths of 884 and $980 \mathrm{mbsl}$, respectively, in February 1995, and stored in CNRS Gif sur Yvette (France). Sampling was done at high resolution (every $2-5 \mathrm{~cm}$ ) throughout the cores and at $1-2 \mathrm{~cm}$ resolution across the sapropels. Core 9509 lies directly beneath the pre-Aswan plume of suspended sediment (the Nile Fan) discharged during the annual Nile flood. Core 9501 was recovered on the continental slope southwest of Cyprus and lies beneath the northern arm of the Eastern Mediterranean anticlockwise surface gyre. Both cores have previously been studied at high resolution, including sediment characteristics, colour index colour parameters, $\delta^{18} \mathrm{O}$ of $G$. ruber, and $\mathrm{Sr}$ isotopes, which gives valuable context for the present study (AlmogiLabin et al., 2009; Box et al., 2011). The complete absence of benthic foraminifera in sapropel S1 in core 9509 suggests anoxic conditions, whereas in core 9501 their occurrence in S1, albeit in very small numbers, indicates more dysaerobic conditions and periods of reventilation (Avnaim-Katav, 2005; Almogi-Labin et al., 2009).

The sedimentary record of core 9509 over the last 86 ka shows the strong influence of the River Nile, as evidenced by higher sedimentation rates compared with the northern 9501 core $(20 \mathrm{~cm} / \mathrm{ka}$ vs. $5 \mathrm{~cm} / \mathrm{ka})$, continuously darker sediment colour, and higher TOC values (0.6-1.9 wt $\%)$ vs. $0.25 \mathrm{wt} \%$ outside sapropels (Almogi-Labin et al., 2009). Increased Nile discharge occurred from 15 to $\sim 5 \mathrm{ka}$ (Revel et al., 2014), coinciding with the African Humid Period (AHP; 11.5-5.5 ka; Adkins et al., 2006; deMenocal et al., 2000) which encompasses sapropel S1. The Sr isotope record of core 9509 suggests that the AHP was marked by increased contribution of White Nile sediment, but decreased Blue Nile flux due to vegetation inhibiting soil erosion in the catchment area (Box et al., 2011). Saharan dust flux at core 9509 was low through the AHP ( $<2 \mathrm{~g} / \mathrm{cm} 2 / \mathrm{ka}$ at $6 \mathrm{ka}$ : Box et al., 2011), reflecting the 'greening of the Sahara' (Larrasoana et al., 2003; Revel et al., 2010).

Scrivner et al. (2004) identified Nile River influence reaching as far as Cyprus (ODP 967) in sapropels S1 and S5 and noted a temporal link between maxima in the Nile discharge and freshwater addition due to enhanced westerly rainfall activity. Sea-land $\delta^{18} \mathrm{O}$ differences $\left(\Delta \delta^{18} \mathrm{O}_{\text {sea- }}\right.$ land) measured at sites 9509 and 9501 by Almogi-Labin et al. (2009) suggest that the 'amount effect' lowered rainfall $\delta^{18} \mathrm{O}$ during interglacial sapropel periods, adding to the seawater $\delta^{18} \mathrm{O}$ decrease brought about by melting of continental ice and the strong effects in the southern Levantine basin of the high Nile River freshwater input.

A brief reventilation event during sapropel $\mathrm{S} 1$ at ca $8.2 \mathrm{ka}$ was identified in the G. ruber $\delta^{18} \mathrm{O}$ record of core 9509 (Almogi-Labin et al., 2009). This reventilation event has been observed in a number of studies of Mediterranean sapropels (e.g. Casford et al., 2001; Hennekam et al., 2014; Filippidi et al., 2016), and is related to the occurrence of a global Holocene 'cold event' (Alley et al., 1997). Filippidi et al. (2016) recently identified a second cool event, dated at 7.4 to $7.2 \mathrm{ka}$, in the Adriatic and Aegean basins.

\subsection{Analytical methods}

The sectioned core samples were brought to the Geological Survey of Israel (GSI) and freeze dried for further analysis. Bulk chemical analyses were made at the GSI, after low temperature ashing $\left(105^{\circ} \mathrm{C}\right)$ and sodium peroxide fusion (sintering) (Merck 95\%), using Inductively Coupled Plasma Optical Emission Spectrometry (ICP-OES; Perkin Elmer Optima 3300) for major element composition and Inductively Coupled Plasma Mass Spectrometry (ICP-MS; Perkin Elmer DRII Sciex) for trace element composition. Analytical errors are $\pm 5 \%$. Aluminum was measured to normalize elemental ratios (Thomson et al., 1999) and to calculate molybdenum and uranium enrichment factors $\left(\mathrm{Mo}_{\mathrm{EF}}\right.$ and $\left.\mathrm{U}_{\mathrm{EF}}\right)$ where $\mathrm{X}_{\mathrm{EF}}=\left[(\mathrm{X} / \mathrm{Al})_{\text {sample }} /(\mathrm{X} / \mathrm{Al})_{\text {average shale }}\right]$ (Tribovillard et al., 2006). Total Organic Carbon (TOC) was measured after acidification using TOC-VCPN Shimadzu TOC analyser. Scanning electron microscope (SEM) imaging of Fe minerals were done on an FEI Quanta 450 SEM at the GSI.

Sample digestion and column chemistry for isotopic analysis were done in the clean laboratory at the Hebrew University of Jerusalem (HUJI). Samples were pyrolized at $800{ }^{\circ} \mathrm{C}$ for approximately $12 \mathrm{~h}$ to remove organic carbon. The pyrolized residue was then dissolved using 
$\mathrm{HF}, \mathrm{HNO}_{3}$ and $\mathrm{HCl}$ in the presence of $\mathrm{H}_{2} \mathrm{O}_{2}$. Molybdenum separation for isotopic analysis was done using the chromatographic column procedure of Archer and Vance $(2004,2008)$, which sequentially elutes matrix elements, $\mathrm{Cu}, \mathrm{Fe}$ and Mo. Prior to column chemistry, $1 \mathrm{ml}$ of precleaned anion exchange resin (Biorad anion resin AG MP-1 m, 100-200 mesh, chloride form) was loaded into $10 \mathrm{ml}$ plastic Muromac ${ }^{\circledR}$ columns. After further on-column resin cleaning, the sample was loaded with $7 \mathrm{M}$ $\mathrm{HCl}$ containing $0.01 \%$ of $30 \% \mathrm{H}_{2} \mathrm{O}_{2}$. Iron was eluted with $2 \mathrm{M} \mathrm{HCl}$ and Mo with $0.5 \mathrm{M} \mathrm{HNO}_{3}$. Following elution, solutions were centrifuged to remove any particle contaminants, fluxed three times with $0.5 \mathrm{ml}$ concentrated $\mathrm{HNO}_{3}$ and dried.

Molybdenum isotopic analyses were carried out at the University of Bristol, using a Thermo Finnegan Neptune instrument. Solutions were introduced into the mass spectrometer in a $2 \%(\mathrm{v} / \mathrm{v}) \mathrm{HNO}_{3}$ solution by means of a CPI (The Netherlands) PFA nebulizer and spray chamber fitted to a CETAC Aridus. Mass discrimination correction was achieved using the double spike as described previously by others for Mo (Siebert et al., 2001), and using the specific procedure previously described for Zn (Bermin et al., 2006). All mass spectrometric errors are propagated through the double-spike calculation to obtain the final analytical uncertainty. Details of standards, calibration procedures and errors are given in Azrieli-Tal et al. (2014). All Mo isotope compositions for samples are reported relative to NIST SRM $3134 \delta^{98 / 95}$ Mo $=0.25 \%$ $\left(\delta^{98 / 95}\right.$ Mo seawater - 2.34\%o) (Nägler et al., 2014). Complete replicates of selected sapropel samples (digestion, column chemistry, MC-ICP-MS) were reproducible within $0.10 \%$ (2 SD).

Iron isotope analyses were measured on the Nu Plasma II MC-ICPMS at GSI, using the sample-standard bracketing approach in medium resolution mode. The measurements were made on the 16 samples of the Fe solution fraction extracted during the Mo column chemistry, after a second column chemistry purification stage specifically aimed at Fe isotopes. Since these solutions contain a large excess of Fe relative to the amount required for Fe isotope analysis, concentrations were adjusted by evaporating to dryness, redissolving in $6 \mathrm{M} \mathrm{HCl}$ and taking the appropriate amount for MC-ICP-MS. The same $10 \mathrm{ml}$ columns containing about $1 \mathrm{ml}$ AG 1-x4 resin (200-400 mesh, Cl form, Bio-Rad) were used for the chromatographic purification. Sample loading and matrix elution were performed with $6 \mathrm{M} \mathrm{HCl}$ and Fe collection with $1 \mathrm{M}$ $\mathrm{HCl}$.

The $\delta^{56} \mathrm{Fe}$ values are reported relative to the IRMM-014 metal standard. Results for repeated $\delta^{56} \mathrm{Fe}$ measurements of the ETH Fe-salt solution (over 18 months) are $-0.73 \pm 0.11 \%$ ( $2 \mathrm{SD}, \mathrm{n}=61$ ) identical within errors to previously published data $(-0.71 \pm 0.18 \%$, Teutsch et al., 2009 and $-0.73 \pm 0.10 \%$, Fehr et al., 2008). The geochemical reference samples IF-G processed in four different sessions (including digestion and column chemistry) produced $\delta^{56} \mathrm{Fe}=0.63 \pm 0.09 \%$ ( $\left.2 \mathrm{SD}, \mathrm{n}=16\right)$ in excellent agreement with the recommended value of $0.639 \pm 0.013 \%$ (Craddock and Dauphas, 2011 and references cited therein).

All published $\delta^{56} \mathrm{Fe}$ analyses cited relative to the Madison igneous rock standard (Beard et al., 2003b) are adjusted in this paper to the IRMM-014 standard. Data reported using $\delta^{57} \mathrm{Fe}$ IRMM-014 values (Azrieli-Tal et al., 2014) are presented as $\delta^{56} \mathrm{Fe}$ values using the mass fractionation relationship. Isotopic fractionation factors are expressed as $\Delta_{\mathrm{A}-\mathrm{B}}=\delta_{\mathrm{A}}-\delta_{\mathrm{B}}$.

The sequential $\mathrm{Fe}$ speciation studies provide a measure of highly reactive $\mathrm{Fe}\left(\mathrm{Fe}_{\mathrm{HR}}\right)$, which refers to $\mathrm{Fe}$ minerals that are considered highly reactive towards biological/abiological reduction under anoxic conditions (Canfield et al., 1992; Canfield et al., 1996; Raiswell and Canfield, 1998; Poulton et al., 2004). Fe extractions were made at the University of Leeds using the calibrated extraction of Poulton and Canfield (2005), which has recently been modified for application to recent sediments (see Goldberg et al., 2012; Zegeye et al., 2012). The first step consists of a $0.5 \mathrm{~N} \mathrm{HCl}$ extraction for $1 \mathrm{~h}$, which targets reduced $\mathrm{Fe}$ phases, including acid volatile $\mathrm{Fe}$ sulphides $\left(\mathrm{Fe}_{\mathrm{Avs}}\right)$ such as mackinawite, surface-reduced $\mathrm{Fe}(\mathrm{II})$, and potentially some poorly crystalline ferrous carbonate/phosphate phases. The $\mathrm{Fe}_{\mathrm{AVs}}$ is also measured separately (see below), and thus after subtraction of $\mathrm{Fe}_{\mathrm{Avs}}$, this pool consists of the remaining non-sulphidized Fe(II) minerals (termed $\mathrm{Fe}(\mathrm{II})_{\mathrm{HCl}}$ ). The $\mathrm{Fe}(\mathrm{II})_{\mathrm{HCl}}$ pool was determined by spectrophotometer using the ferrozine assay (Stookey, 1970), immediately after completion of the extraction. The $0.5 \mathrm{~N} \mathrm{HCl}$ extraction also extracts the most reactive $\mathrm{Fe}(\mathrm{III})$ minerals remaining in the sediment, in particular ferrihydrite (termed $\left.\mathrm{Fe}(\mathrm{III})_{\mathrm{HCl}}\right)$. This pool was determined by atomic adsorption spectrometry (AAS), after subtraction of $\mathrm{Fe}(\mathrm{II})_{\mathrm{HCl}}$. The second sequential extraction utilizes a buffered Na dithionite solution to extract more crystalline Fe (oxyhydr)oxide minerals such as goethite and hematite (termed $\mathrm{Fe}_{\text {ox }}$; Poulton and Canfield, 2005). The final sequential extraction targets magnetite, using a Na oxalate extraction (termed $\mathrm{Fe}_{\text {mag; }}$; Poulton and Canfield, 2005). The $\mathrm{Fe}_{\mathrm{ox}}$ and $\mathrm{Fe}_{\text {mag }}$ fractions were measured via AAS, with all sequential extraction steps giving a RSD of $<5 \%$, based on replicate extractions.

Pyrite $\left(\mathrm{Fe}_{\mathrm{py}}\right)$ and $\mathrm{Fe}_{\mathrm{Avs}}$ were determined via the procedure of Canfield et al. (1986), whereby $\mathrm{Fe}_{\mathrm{Avs}}$ is extracted using hot $6 \mathrm{~N} \mathrm{HCl}$, and $\mathrm{Fe}_{\mathrm{py}}$ is extracted from the residue via chromous chloride distillation. These determinations indicated that $\mathrm{Fe}_{\mathrm{Avs}}$ was below detection limits. The $\mathrm{H}_{2} \mathrm{~S}$ liberated by chromous chloride distillation was precipitated as $\mathrm{Ag}_{2} \mathrm{~S}$, and sulphide was determined gravimetrically and then converted to $\mathrm{Fe}$ concentrations according to the stoichiometry of the mineral phase (i.e. as $\mathrm{FeS}_{2}$ for pyrite). Replicate extractions gave a RSD of $<5 \%$ for the sulphide extraction. The sum of $\mathrm{Fe}(\mathrm{II})_{\mathrm{HCl}}, \mathrm{Fe}$ (III) $\mathrm{HCl}_{1}, \mathrm{Fe}_{\mathrm{ox}}, \mathrm{Fe}_{\mathrm{mag}}$, and $\mathrm{Fe}_{\mathrm{py}}$ defines the $\mathrm{Fe}_{\mathrm{HR}}$ pool. Total $\mathrm{Fe}\left(\mathrm{Fe}_{\mathrm{T}}\right)$ values were taken from the bulk chemical composition determined by sintering procedure and ICP-OES analysis.

Box et al. (2011) and Azrieli-Tal et al. (2014) analysed the $<63 \mu \mathrm{m}$ size fraction, which eliminates the foraminiferal contribution, to provide a representative geochemical record of the metallic elements in sapropel S1. Here, we measured the trace and major element and Mo isotope composition of both the bulk sediment and $<63 \mu \mathrm{m}$ fractions for core 9509. The close proximity to the Nile suspended load with its high detrital mineral content might lead to mineralogical and geochemical differences between the fine and coarse-grained material in the sapropel, and it was considered advantageous to measure both fractions. Only the bulk sediment fraction was measured for core 9501.

Table 1 lists sample depths, and elemental and isotopic data used in this study as proxies (elemental data are normalized to $\mathrm{Al}$ with the exception of S and TOC). A complete list of all measured concentration data is provided in Supplementary data Table A1. Fe speciation data are presented in Table 2 .

\section{Results}

\subsection{Chemical depth profiles}

\subsubsection{Productivity and major element profiles in core 9509}

The typically dark colouration sediments from core 9509 preclude assignment of sapropel boundaries on the basis of colour (Almogi-Labin et al., 2009). Thus, following Thomson et al. (1999) sapropel boundaries are based on increases in TOC $\mathrm{wt}_{\mathrm{t}} \mathrm{\%}$ and $\mathrm{Ba} / \mathrm{Al}$ (a proxy for export paleoproductivity) values above background sedimentary values. The TOC wt $\%$ and $\mathrm{Ba} / \mathrm{Al}$ depth profiles (Figs. 2a, b; Table 1) show marked increases in samples between 113 and $173 \mathrm{~cm}$, which we use to assign the sapropel boundaries. The concurrence of these boundaries for both proxies indicates that commonly observed post-sapropel reventilation and consequent TOC 'burn down' did not occur (cf. Thomson et al., 1999; de Lange et al., 2008). Using the age model of Almogi-Labin et al. (2009), the sapropel period defined by the $\mathrm{Ba} / \mathrm{Al}$ enrichment is 9.7 to $6.9 \mathrm{ka}$. The sapropel TOC values reach $1.6 \%$ and are therefore significantly lower than TOC values in coeval sediments deposited in deeper water ( $\sim 4 \mathrm{wt} \%$ in core $967 \mathrm{D}$; Vance et al., 2004). In the following presentation, the locations of samples are defined by their average depth in $\mathrm{cm}$; the corresponding age in ka will be given in the 
Table 1

Sample, elemental and isotopic data used in this study.

\begin{tabular}{|c|c|c|c|c|c|c|c|c|c|c|c|c|c|c|c|c|c|c|}
\hline Sample no & $\begin{array}{l}\text { Base/top } \\
(\mathrm{cm})\end{array}$ & $\begin{array}{l}\text { Mean } \\
\text { depth } \\
(\mathrm{cm})\end{array}$ & $\begin{array}{l}\mathrm{Ba} / \mathrm{Al} \\
(\mathrm{g} / \mathrm{g} \\
\left.10^{4}\right)\end{array}$ & $\begin{array}{l}\text { TOC } \\
\text { (wt } \\
\% \text { ) }\end{array}$ & S (wt $\%)$ & $\begin{array}{l}\mathrm{Fe} / \\
\mathrm{Al} \\
(\mathrm{g} / \\
\mathrm{g})\end{array}$ & $\begin{array}{l}\mathrm{Ti} / \mathrm{Al} \\
(\mathrm{g} / \mathrm{g})\end{array}$ & $\begin{array}{l}\mathrm{Mn} / \\
\mathrm{Al}(\mathrm{g} / \\
\mathrm{g} \\
\left.{ }^{1} 10^{4}\right)\end{array}$ & $\begin{array}{l}\mathrm{Mo} / \\
\mathrm{Al}(\mathrm{g} / \\
\mathrm{g} \\
\left.{ }^{1} 10^{4}\right)\end{array}$ & $\mathrm{Mo}_{\mathrm{EF}}$ & $\begin{array}{l}\mathrm{U} / \mathrm{Al} \\
(\mathrm{g} / \mathrm{g} \\
\left.10^{4}\right)\end{array}$ & $\mathrm{U}_{\mathrm{EF}}$ & $\begin{array}{l}\mathrm{V} / \mathrm{Al} \\
(\mathrm{g} / \mathrm{g} \\
\left.10^{4}\right)\end{array}$ & $\begin{array}{l}\mathrm{Sb} / \mathrm{Al} \\
(\mathrm{g} / \mathrm{g} \\
\left.10^{4}\right)\end{array}$ & $\begin{array}{l}\delta^{56} \mathrm{Fe} \\
\text { (IRMM- } \\
14 \% 0 \text { ) }\end{array}$ & $\begin{array}{l}2 \sigma \\
\text { error }^{a}\end{array}$ & $\begin{array}{l}\delta^{98 / 95} \text { Mo } \\
\text { (SRM- } \\
3134 \% \text { ) })^{\text {a }}\end{array}$ & $\begin{array}{l}2 \sigma \\
\text { error }^{\mathrm{a}}\end{array}$ \\
\hline \multicolumn{19}{|c|}{ Core 9509 bulk samples } \\
\hline 1341 & $5 / 7$ & 6 & 22 & & & 0.81 & 0.094 & 135 & 0.15 & 1.24 & 0.36 & 3.31 & 16.50 & 0.08 & & & -0.07 & 0.02 \\
\hline 1342 & $40 / 42$ & 41 & 21 & & & 0.77 & 0.088 & 131 & 0.13 & 1.13 & 0.31 & 2.87 & 15.68 & 0.07 & & & -0.07 & 0.03 \\
\hline 1297 & $57 / 59$ & 58 & 26 & 0.56 & 0.38 & 0.80 & 0.076 & 135 & 0.44 & 3.67 & 0.68 & 6.31 & 21.25 & 0.25 & 0.08 & 0.04 & 0.10 & 0.05 \\
\hline 1343 & $80 / 82$ & 81 & 19 & & & 0.81 & 0.085 & 150 & 0.16 & 1.34 & 0.38 & 3.55 & 15.35 & 0.08 & & & 0.14 & 0.03 \\
\hline 1298 & $97 / 99$ & 98 & 25 & 0.62 & 0.26 & 0.78 & 0.081 & 162 & 0.19 & 1.57 & 0.45 & 4.17 & 15.63 & 0.12 & 0.11 & 0 & 0.64 & 0.03 \\
\hline 1299 & $107 / 109$ & 108 & 25 & 0.57 & 0.29 & 0.76 & 0.079 & 116 & 0.23 & 1.94 & 0.49 & 4.50 & 15.16 & 0.12 & -0.03 & 0.05 & 1.07 & 0.05 \\
\hline 1300 & $112 / 114$ & 113 & 28 & 0.58 & 0.25 & 0.75 & 0.078 & 126 & 0.29 & 2.45 & 0.54 & 4.95 & 15.06 & 0.12 & $\begin{array}{l}0.07 \\
(-0.04)\end{array}$ & $\begin{array}{l}0.04 \\
(0.02)\end{array}$ & 0.83 & 0.05 \\
\hline 1301 & 116,120 & 118 & 47 & 1.00 & 0.65 & 0.89 & 0.107 & 175 & 0.53 & 4.46 & 0.90 & 8.29 & 18.50 & 0.29 & 0.04 & 0.14 & 0.82 & 0.05 \\
\hline 1302 & $120 / 124$ & 122 & 68 & 0.97 & 1.22 & 0.93 & 0.102 & 139 & 1.60 & 13.44 & 1.43 & 13.21 & 21.41 & 0.62 & $\begin{array}{l}0.01 \\
(0.00)\end{array}$ & $\begin{array}{l}0.05 \\
(0.05)\end{array}$ & 0.55 & 0.05 \\
\hline 1303 & $124 / 128$ & 126 & 80 & 1.13 & 1.14 & 0.77 & 0.077 & 170 & 1.79 & 15.05 & 1.63 & 15.04 & 24.61 & 0.78 & 0.03 & 0.06 & 0.56 & 0.05 \\
\hline 1304 & $128 / 132$ & 130 & 73 & 0.99 & 1.09 & 0.75 & 0.078 & 174 & 1.41 & 11.84 & 1.30 & 12.04 & 25.95 & 0.60 & 0.02 & 0.03 & $\begin{array}{l}0.50 \\
(0.56)^{*}\end{array}$ & $\begin{array}{l}0.03 \\
(0.06)\end{array}$ \\
\hline 1305 & $132 / 136$ & 134 & 53 & 0.66 & 1.01 & 0.74 & 0.079 & 226 & 1.22 & 10.22 & 1.14 & 10.50 & 18.04 & 0.31 & $\begin{array}{l}0.04 \\
(0.01)\end{array}$ & $\begin{array}{l}0.09 \\
(0.07)\end{array}$ & $\begin{array}{l}0.76 \\
(0.70)^{\text {* }}\end{array}$ & $\begin{array}{l}0.03 \\
(0.03)\end{array}$ \\
\hline 1306 & $136 / 140$ & 138 & 58 & 0.69 & 1.09 & 0.92 & 0.110 & 190 & 1.23 & 10.37 & 1.18 & 10.93 & 20.49 & 0.34 & & & 0.62 & 0.03 \\
\hline 1307 & $140 / 143$ & 141.5 & 51 & 0.90 & 0.90 & 0.75 & 0.080 & 162 & 2.06 & 17.31 & 1.17 & 10.84 & 20.35 & 1.36 & -0.03 & 0.05 & 0.41 & 0.03 \\
\hline 1308 & $145 / 148$ & 146.5 & 57 & 1.58 & 1.03 & 0.91 & 0.109 & 164 & 2.13 & 17.92 & 1.09 & 10.10 & 20.05 & 1.36 & 0.01 & 0.03 & 0.28 & 0.05 \\
\hline 1309 & $148 / 151$ & 149.5 & 68 & 1.08 & 1.13 & 0.91 & 0.106 & 137 & 1.78 & 14.97 & 1.07 & 9.87 & 20.25 & 0.46 & 0.03 & 0.02 & 0.28 & 0.05 \\
\hline 1310 & $151 / 154$ & 152.5 & 66 & 1.09 & 1.22 & 0.90 & 0.099 & 136 & 1.84 & 15.46 & 1.05 & 9.73 & 19.26 & 0.47 & 0.02 & 0.08 & 0.59 & 0.24 \\
\hline 1311 & $154 / 157$ & 155.5 & 93 & 1.32 & 1.04 & 0.98 & 0.115 & 250 & 3.27 & 27.46 & 1.60 & 14.80 & 26.37 & 0.84 & 0.08 & 0.06 & $\begin{array}{l}0.16 \\
(0.58)\end{array}$ & $\begin{array}{l}0.05 \\
(0.03)\end{array}$ \\
\hline 1312 & $157 / 160$ & 158.5 & 66 & 1.25 & 1.57 & 0.90 & 0.104 & 119 & 1.62 & 13.58 & 1.22 & 11.24 & 20.52 & 0.53 & 0.05 & 0.03 & 0.76 & 0.05 \\
\hline 1313 & $160 / 164$ & 162 & 62 & 1.29 & 1.18 & 0.73 & 0.077 & 164 & 1.71 & 14.38 & 1.11 & 10.23 & 29.59 & 0.67 & 0.02 & 0.06 & 0.53 & 0.05 \\
\hline 1314 & $164 / 168$ & 166 & 54 & 1.18 & 1.57 & 0.88 & 0.078 & 128 & 2.17 & 18.19 & 1.03 & 9.49 & 26.65 & 0.46 & 0.01 & 0.05 & 0.83 & 0.05 \\
\hline 1315 & $172 / 174$ & 173 & 31 & 0.63 & 1.08 & 0.84 & 0.084 & 525 & 0.91 & 7.61 & 0.56 & 5.18 & 16.71 & 0.13 & & & 0.20 & 0.03 \\
\hline 1316 & $177 / 179$ & 178 & 29 & 0.55 & 0.52 & 0.77 & 0.085 & 624 & 0.31 & 2.59 & 0.46 & 4.27 & 17.00 & 0.13 & -0.05 & 0.03 & 0.69 & 0.03 \\
\hline 1317 & $187 / 187$ & 188 & 30 & 0.52 & 0.34 & 0.81 & 0.089 & 219 & 0.21 & 1.75 & 0.45 & 4.11 & 17.75 & 0.12 & & & 0.70 & 0.05 \\
\hline 1318 & $217 / 219$ & 218 & 29 & 0.51 & 0.38 & 0.82 & 0.096 & 181 & 0.18 & 1.55 & 0.35 & 3.26 & 17.87 & 0.09 & & & 0.43 & 0.05 \\
\hline 1344 & $230 / 232$ & 231 & 20 & & 0.28 & 0.76 & 0.081 & 139 & 0.20 & 1.72 & 0.29 & 2.66 & 14.66 & 0.07 & & & 0.31 & 0.03 \\
\hline 1345 & $260 / 262$ & 261 & 29 & & & 0.83 & 0.096 & 188 & 0.19 & 1.61 & 0.49 & 4.49 & 13.27 & 0.06 & & & 0.17 & 0.03 \\
\hline 1346 & $270 / 272$ & 271 & 46 & & & 0.82 & 0.116 & 336 & 0.33 & 2.77 & 0.69 & 6.36 & 21.05 & 0.10 & & & 0.17 & 0.03 \\
\hline 1347 & $310 / 312$ & 311 & 70 & & & 0.83 & 0.107 & 228 & 0.06 & 0.50 & 0.10 & 0.90 & 5.95 & 0.03 & & & & \\
\hline \multicolumn{19}{|c|}{$9509<63 \mu \mathrm{m}$ size fraction } \\
\hline $1299 b$ & $107 / 109$ & 108 & & & & & & & & & & & & & & & 0.50 & 0.02 \\
\hline $1301 b$ & $116 / 120$ & 118 & 42 & & 0.27 & 0.71 & 0.072 & 154 & 0.40 & 7.90 & 0.94 & 8.68 & 18.61 & 0.24 & & & & \\
\hline $1302 b$ & $120 / 124$ & 122 & 63 & & 0.70 & 0.75 & 0.071 & 136 & 1.08 & 11.96 & 1.42 & 13.15 & 21.33 & 0.51 & & & 0.24 & 0.03 \\
\hline $1303 b$ & $124 / 128$ & 126 & 67 & & 0.72 & 0.73 & 0.072 & 132 & 0.97 & 12.12 & 1.44 & 13.32 & 22.00 & 0.57 & & & & \\
\hline $1304 b$ & $128 / 132$ & 130 & 66 & & 0.72 & 0.71 & 0.072 & 148 & 0.82 & 10.39 & 1.24 & 11.42 & 24.18 & 0.43 & & & & \\
\hline $1305 b$ & $132 / 136$ & 134 & 49 & & 0.57 & 0.70 & 0.073 & 208 & 0.67 & 8.53 & 1.02 & 9.38 & 16.26 & 0.22 & & & & \\
\hline $1306 \mathrm{~b}$ & $136 / 140$ & 138 & 50 & & 0.53 & 0.70 & 0.074 & 176 & 0.82 & 9.82 & 1.17 & 10.80 & 17.94 & 0.27 & & & & \\
\hline $1307 \mathrm{~b}$ & $140 / 143$ & 141.5 & 47 & & 0.52 & 0.75 & 0.075 & 158 & 1.43 & 9.15 & 1.09 & 10.06 & 19.65 & 0.31 & & & 0.17 & 0.03 \\
\hline $1308 b$ & $145 / 148$ & 146.5 & 52 & & 0.67 & 0.72 & 0.073 & 135 & 1.02 & 8.43 & 1.00 & 9.27 & 18.72 & 0.33 & & & & \\
\hline $1309 b$ & $148 / 151$ & 149.5 & 61 & & 0.72 & 0.71 & 0.071 & 128 & 1.23 & 8.32 & 0.99 & 9.14 & 18.20 & 0.41 & & & & \\
\hline $1310 \mathrm{~b}$ & $151 / 154$ & 152.5 & 60 & & 0.66 & 0.69 & 0.071 & 132 & 1.28 & 8.70 & 1.04 & 9.57 & 19.28 & 0.41 & & & $\begin{array}{l}0.07 \\
(0.06)^{*}\end{array}$ & $\begin{array}{l}0.015 \\
(0.03)\end{array}$ \\
\hline $1311 b$ & $154 / 157$ & 155.5 & 71 & & 0.88 & 0.71 & 0.072 & 163 & 1.47 & 9.73 & 1.16 & 10.69 & 20.00 & 0.52 & & & & \\
\hline $1312 b$ & $157 / 160$ & 158.5 & 63 & & 0.73 & 0.72 & 0.073 & 113 & 1.05 & 9.72 & 1.16 & 10.68 & 19.46 & 0.46 & & & 0.47 & 0.15 \\
\hline $1313 b$ & $160 / 164$ & 162 & 56 & & 0.66 & 0.71 & 0.073 & 146 & 1.24 & 10.22 & 1.22 & 11.24 & 31.74 & 0.61 & & & & \\
\hline $1314 b$ & $164 / 168$ & 166 & & & & & & & & & & & & & & & 0.70 & 0.03 \\
\hline \multicolumn{19}{|c|}{ Core 9501 bulk samples } \\
\hline 1256 & $64 / 66$ & 65 & 30 & & & 0.81 & 0.075 & 463 & 0.73 & 6.1 & 0.22 & 2.03 & 18.74 & 0.15 & & & -0.04 & 0.03 \\
\hline 1257 & $68 / 70$ & 69 & 32 & & & 0.79 & 0.073 & 886 & 1.22 & 10.2 & 0.23 & 2.17 & 17.29 & 0.23 & & & & \\
\hline 1258 & $70 / 72$ & 71 & 33 & & & 0.80 & 0.074 & 661 & 0.84 & 7.0 & 0.22 & 2.03 & 18.08 & 0.18 & & & 0.00 & 0.05 \\
\hline 1259 & $75 / 75$ & 77.5 & 58 & 0.17 & & 0.89 & 0.074 & 1471 & 1.78 & 14.9 & 0.28 & 2.57 & 20.03 & 0.25 & & & 0.40 & 0.05 \\
\hline 1260 & $80 / 85$ & 82.5 & 77 & 0.53 & 0.28 & 0.81 & 0.057 & 89 & 0.43 & 3.6 & 0.39 & 3.56 & 23.97 & 0.22 & & & 0.82 & 0.05 \\
\hline 1261 & $85 / 88$ & 86.5 & 93 & 0.59 & 0.56 & 0.84 & 0.068 & 96 & 0.51 & 4.3 & 0.72 & 6.65 & 32.65 & 0.36 & & & 1.04 & 0.08 \\
\hline 1262 & $88 / 90$ & 89 & 99 & 0.55 & 0.52 & 0.83 & 0.068 & 105 & 0.64 & 5.4 & 0.90 & 8.28 & 36.29 & 0.47 & & & $\begin{array}{l}0.92 \\
(0.71)^{*}\end{array}$ & $\begin{array}{l}0.03 \\
(0.05)\end{array}$ \\
\hline 1263 & $90 / 94$ & 92 & 60 & 0.34 & 0.64 & 0.84 & 0.068 & 133 & 0.69 & 5.8 & 1.04 & 9.63 & 29.30 & 0.58 & & & 0.38 & 0.04 \\
\hline 1264 & $94 / 96$ & 95 & 44 & 0.22 & 0.40 & 0.80 & 0.074 & 151 & 0.61 & 5.1 & 1.14 & 10.51 & 21.67 & 0.55 & & & -0.83 & 0.03 \\
\hline 1265 & $106 / 108$ & 107 & 37 & 0.18 & & 0.78 & 0.076 & 152 & 0.18 & 1.5 & 1.13 & 10.41 & 20.35 & 0.24 & & & 0.39 & 0.03 \\
\hline 1266 & $112 / 114$ & 113 & 41 & 0.18 & & 0.78 & 0.080 & 163 & 0.20 & 1.7 & 1.01 & 9.33 & 25.51 & 0.25 & & & 1.25 & 0.03 \\
\hline
\end{tabular}

${ }^{a}$ Duplicate analyses are indicated in brackets after the first analysis. Duplicate samples denoted by a star were run through a separate full chemistry including digestion. Other duplicates were run through a separate column chemistry only. 
Table 2

Fe speciation data for core 9509 samples.

\begin{tabular}{|c|c|c|c|c|c|c|c|c|c|c|c|c|c|}
\hline Sample no & Bottom (cm) & Top $(\mathrm{cm})$ & $\begin{array}{l}\text { Mean depth } \\
(\mathrm{cm})\end{array}$ & $\begin{array}{l}\mathrm{Fe}(\mathrm{II})_{\mathrm{HCl}} \\
(\mathrm{wt} \%)\end{array}$ & $\begin{array}{l}\mathrm{Fe}(\mathrm{III})_{\mathrm{HCl}} \\
(\mathrm{wt} \%)\end{array}$ & $\begin{array}{l}\mathrm{Fe}_{\mathrm{ox}}(\mathrm{wt} \\
\%)\end{array}$ & $\begin{array}{l}\mathrm{Fe}_{\text {mag }}(\mathrm{wt} \\
\%)\end{array}$ & $\begin{array}{l}\mathrm{Fe}_{\text {py }}(w t \\
\%)\end{array}$ & $\begin{array}{l}\mathrm{Fe}_{\mathrm{HR}}(\mathrm{wt} \\
\%)\end{array}$ & $\mathrm{Fe}_{\mathrm{T}}(\mathrm{wt} \%)$ & $\mathrm{Fe}_{\mathrm{HR}} / \mathrm{FeT}$ & $\mathrm{Fe}_{\mathrm{py}} / \mathrm{Fe}_{\mathrm{HR}}$ & $\begin{array}{l}\text { Corr } \mathrm{Fe}_{\mathrm{py}} / \\
\mathrm{Fe}_{\mathrm{HR}}{ }^{\mathrm{a}}\end{array}$ \\
\hline 1297 & 59 & 57 & 58 & 0.020 & 0.339 & 1.375 & 0.406 & 0.060 & 2.199 & 4.720 & 0.47 & 0.03 & 0.18 \\
\hline 1298 & 99 & 97 & 98 & 0.019 & 0.302 & 1.307 & 0.397 & 0.079 & 2.105 & 6.030 & 0.35 & 0.04 & 0.18 \\
\hline 1300 & 114 & 112 & 113 & 0.021 & 0.264 & 1.233 & 0.360 & 0.085 & 1.963 & 5.670 & 0.35 & 0.04 & 0.18 \\
\hline 1301 & 120 & 116 & 118 & 0.028 & 0.320 & 1.050 & 0.279 & 0.225 & 1.902 & 6.800 & 0.28 & 0.12 & 0.29 \\
\hline 1303 & 128 & 124 & 126 & 0.050 & 0.349 & 0.749 & 0.200 & 0.269 & 1.617 & 4.730 & 0.34 & 0.17 & 0.38 \\
\hline 1304 & 132 & 128 & 130 & 0.039 & 0.343 & 0.736 & 0.194 & & 1.311 & 4.860 & 0.27 & & 0.26 \\
\hline 1305 & 136 & 132 & 134 & 0.027 & 0.299 & 0.806 & 0.225 & 0.275 & 1.633 & 4.890 & 0.33 & 0.17 & 0.35 \\
\hline 1306 & 140 & 136 & 138 & 0.031 & 0.323 & 0.716 & 0.201 & 0.378 & 1.649 & 5.780 & 0.29 & 0.23 & 0.43 \\
\hline 1310 & 151 & 154 & 152.5 & 0.037 & 0.379 & 0.649 & 0.187 & 0.541 & 1.793 & 6.040 & 0.30 & 0.30 & 0.51 \\
\hline 1311 & 157 & 154 & 155.5 & 0.033 & 0.376 & 0.587 & 0.172 & 0.618 & 1.787 & 4.690 & 0.38 & 0.35 & 0.56 \\
\hline 1313 & 164 & 160 & 162 & 0.041 & 0.355 & 0.562 & 0.171 & 0.452 & 1.583 & 4.460 & 0.35 & 0.29 & 0.51 \\
\hline 1315 & 174 & 172 & 173 & 0.026 & 0.333 & 0.958 & 0.242 & 0.302 & 1.861 & 5.480 & 0.34 & 0.16 & 0.34 \\
\hline 1316 & 179 & 177 & 178 & 0.028 & 0.291 & 1.022 & 0.301 & 0.164 & 1.806 & 5.070 & 0.36 & 0.09 & 0.25 \\
\hline 1317 & 189 & 187 & 188 & 0.043 & 0.315 & 1.202 & 0.361 & 0.064 & 1.985 & 5.200 & 0.38 & 0.03 & 0.19 \\
\hline 1318 & 219 & 217 & 218 & 0.046 & 0.312 & 1.213 & 0.405 & 0.119 & 2.095 & 5.770 & 0.36 & 0.06 & 0.21 \\
\hline
\end{tabular}

${ }^{\mathrm{a}}$ Corrected $\mathrm{Fe}_{\mathrm{py}}=\mathrm{Fe}(\mathrm{III})_{\mathrm{HCl}}+\mathrm{Fe}_{\mathrm{py}}$

text and figures where relevant. The low-C non-sapropelic sediment above and below the sapropel layers are referred to as background sediments.

The maximum $\mathrm{Ba} / \mathrm{Al}$ values of $80-100$ are lower than values of $>200$ observed for core ODP-967D at 2550 mbsl (Azrieli-Tal et al., 2014), but are similar to those observed by Tachikawa et al. (2015) in core MDO4-2722 at 1780 mbsl water depth in the central Levantine basin (Fig. 1). A clearly observed depletion in TOC wt $\%$ and $\mathrm{Ba} / \mathrm{Al}$ occurs at $130-134 \mathrm{~cm}(\sim 8.0$ to $8.2 \mathrm{ka}$; Fig. 2$)$. As noted, this depletion (defined by the vertical white band) is recognized in other S1 cores (Casford et al., 2001; Almogi-Labin et al., 2009; Hennekam et al., 2014; Tachikawa et al., 2015) and ascribed to a reventilation event coinciding with the Holocene Cold event at 8.2 ka (Alley et al., 1997). Peak Ba/Al values (at $\sim 155$ and $125 \mathrm{~cm}$ ) occur either side of this reventilation signal, giving a typical double maxima profile, also shown by other proxies discussed below (Fig. 2).

Major element proxies $\mathrm{Fe} / \mathrm{Al}, \mathrm{Ti} / \mathrm{Al}$ and $\mathrm{S}$ wt $\%$ show significantly lower values in the $<63 \mu \mathrm{m}$ fraction relative to the bulk sapropel samples (Fig. 2c, d, e). Both $\mathrm{Fe} / \mathrm{Al}$ and $\mathrm{Ti} / \mathrm{Al}$ show marked rises and falls within the bulk sapropel, compared to lower values in the $<63$ $\mu \mathrm{m}$ fraction. The Fe/Al values of the bulk non-sapropel sediments average at $\sim 0.80$. These are significantly higher than the crustal value of 0.55 (Raiswell and Canfield, 2012), but are typical of Eastern Mediterranean sediments, which are highly influenced by the elevated particulate Fe flux from the Nile River (Poulton and Raiswell, 2002; Lyons and Severmann, 2006). Krom et al. (1999) note that Ti/Al ratios are diagnostic of detrital Nile particulate matter. The good match between the bulk and $<63 \mu \mathrm{m} \mathrm{Fe} / \mathrm{Al}$ and $\mathrm{Ti} / \mathrm{Al}$ data trends in the
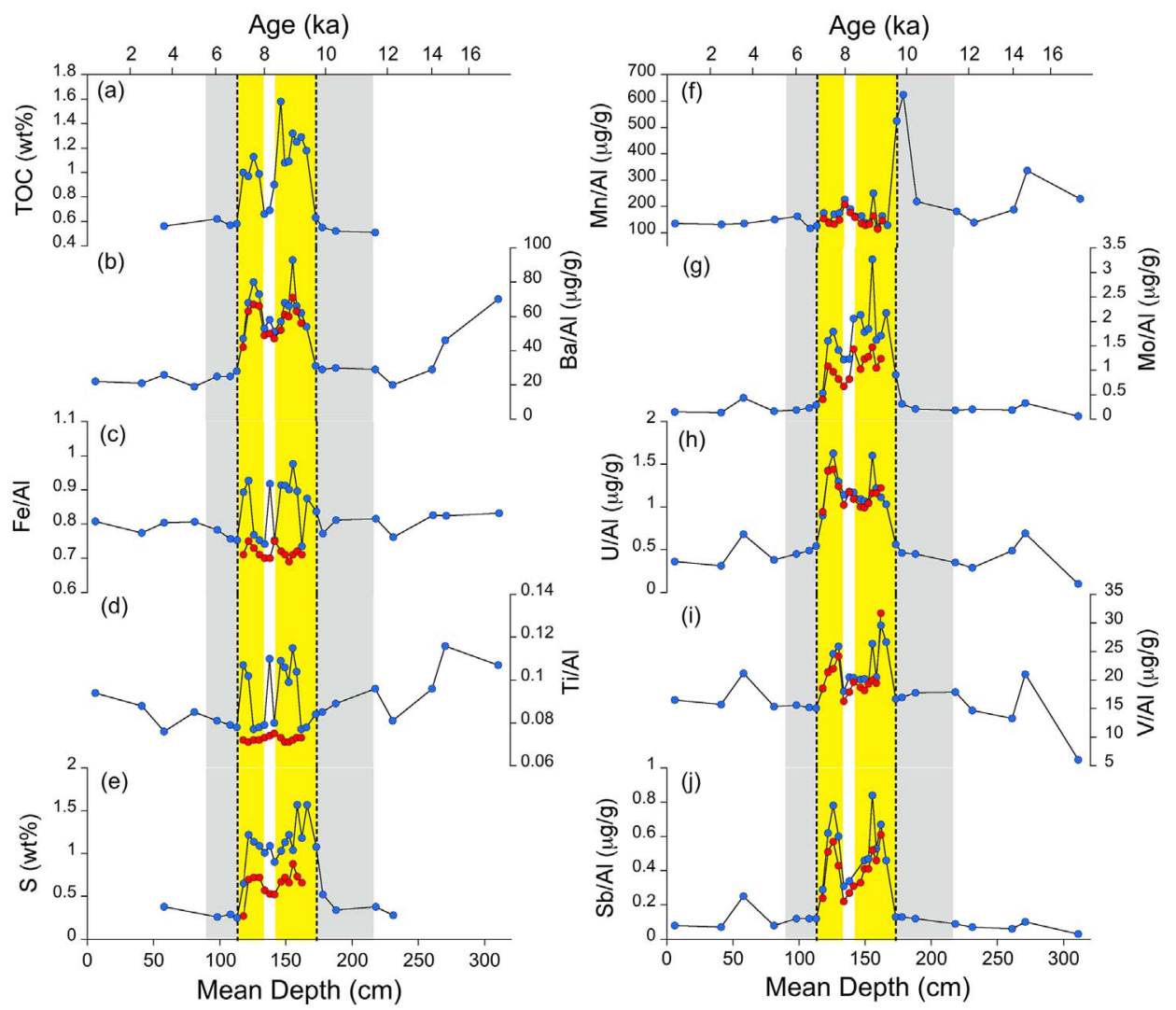

Fig. 2. Productivity, major element and redox sensitive trace element (RSTE) vs. depth profiles for sapropel S1 and enclosing sediments in core 9509. Blue data points indicate bulk samples and red points indicate data for the $<63 \mu \mathrm{m}$ size fraction. Sapropel S1 boundaries defined by Ba enrichment are indicated by the dashed vertical lines and the corresponding extent of TOC enrichment by the yellow shaded area. The grey shaded area indicates the extent of the African Humid Period. The white bar within the sapropel indicates the intra-sapropel ventilation event. The age model for the profile is based on Almogi-Labin et al. (2009). (For interpretation of the references to colour in this figure legend, the reader is referred to the web version of this article.) 
sapropel (Fig. 2c, d) suggests that highly variable $\mathrm{Fe} / \mathrm{Al}$ and $\mathrm{Ti} / \mathrm{Al}$ values in the bulk sapropel could be in part due to the presence of coarsegrained detrital igneous Fe-Ti minerals, such as ilmenite or titanomagnetite, which are retained in the $>63 \mu \mathrm{m}$ fraction. Correspondingly, the drop in $S$ in the $<63 \mu \mathrm{m}$ fraction relative to the bulk sapropel (Fig. 2e) could reflect the retention of $S$ as coarse-grained pyrite framboidal aggregates during sieving, since SEM imaging shows that framboidal pyrite aggregates are often 'nested' within recesses in larger grains of the calcitic clay matrix of the sapropel.

\subsubsection{Redox sensitive trace element proxies in core 9509}

Depth profiles for the set of RSTE proxies (Mn/Al, U/Al, Mo/Al V/Al and $\mathrm{Sb} / \mathrm{Al}$ ) (Fig. 2f-j) show distinct enrichments in the sapropel. There is generally good correspondence between the bulk and $<63 \mu \mathrm{m}$ data, with the notable exception of $\mathrm{Mo} / \mathrm{Al}$, which shows similar trends but lower values in the $<63 \mu \mathrm{m}$ fraction (Fig. $2 \mathrm{~g}$ ). As for $\mathrm{Ba} / \mathrm{Al}$ ratios, RSTE of the same magnitude as in core MD04-2722 (Tachikawa et al., 2015).

The RSTE depth profiles show a response to the 8.2 ka reventilation event with well-defined depletions leading to the previously noted characteristic double maxima form of the sapropel profiles (Fig. $2 \mathrm{~g}-\mathrm{j}$ ). The Mo and $\mathrm{V}$ maxima are higher in the lower (earlier) part of the sapropel (152-162 cm; 9.1-9.2 ka), possibly reflecting weaker recovery of the sapropel after the ventilation event (also reflected by lower TOC in the upper part of the sapropel; Fig. 2a). The Mn/Al profile also shows a small peak during the reventilation event, consistent with $\mathrm{MnO}_{\mathrm{x}}$ mineral precipitation. However, unusually in terms of other studies of S1 sapropels (e.g., Thomson et al., 1999; de Lange et al., 2008), Mn/Al does not show a significant peak at the upper boundary. This is consistent with the absence of post-sapropel oxidation, previously noted in the TOC and $\mathrm{Ba} / \mathrm{Al}$ boundaries (Fig. 2). An additional distinct feature of the $\mathrm{Mn} / \mathrm{Al}$ profile is the large peak at $173-178 \mathrm{~cm}$ immediately preceding sapropel formation. This peak was interpreted to reflect mobilization of $\mathrm{Mn}$ in anoxic sediment during S1, which reprecipitated within oxic sediment beneath the organic-rich layer (Van Santvoort et al., 1996).

Notably, productivity, major element and RSTE proxies do not show a significant rise with the onset of the AHP (Fig. 2); throughout nonsapropel times during the AHP, proxy values are approximately the same as for almost all post sapropel sediments. On the other hand, elevated $\mathrm{Ti} / \mathrm{Al}$ ratios (relative to baseline values of $\sim 0.09$ in post-sapropel sediments) are found in pre-sapropel sediments at 17-14 ka (Fig. 2d). Slight increases in $\mathrm{Ba} / \mathrm{Al}, \mathrm{Mn} / \mathrm{Al}, \mathrm{U} / \mathrm{Al}, \mathrm{V} / \mathrm{Al}$ are also observed during the 16-14 ka period.

\subsection{Geochemical profiles in core 9501}

The lower resolution proxy elemental data for core 9501, located on the continental slope $380 \mathrm{~km}$ north of core 9509 (Fig. 1), show different patterns from core 9509 (Fig. 3). The upper sapropel boundary of core 9501 at $74 \mathrm{~cm}$ (defined by Ba/Al ratios) indicates that the $\mathrm{S} 1$ event had terminated by ca $7.6 \mathrm{ka}$, The narrower zone of TOC enrichment $(\sim 80-92 \mathrm{~cm})$ compared to $\mathrm{Ba} / \mathrm{Al}$ enrichment also indicates that ventilation oxidized the upper $6 \mathrm{~cm}$ of the sapropel. Termination of sapropel $\mathrm{S} 1$ is also recorded by $\mathrm{Mn} / \mathrm{Al}$ and $\mathrm{Fe} / \mathrm{Al}$ peaks at the TOC boundary. It thus appears that, unlike core 9509, the weakening and cessation of sapropel S1 formation at site 9501 is closely associated in time with the 8.2 ka reventilation event.

The $\mathrm{Ba} / \mathrm{Al}, \mathrm{Fe} / \mathrm{Al} \mathrm{S}$ wt $\%$ and $\mathrm{V} / \mathrm{Al}$ show enrichments in the early sapropel, whereas $\mathrm{U} / \mathrm{Al}$ and $\mathrm{Mo} / \mathrm{Al}$ trends are significantly different from those of core 9509 sapropel S1: Mo/Al does not appreciably rise in the sapropel, but peaks near the upper boundary, whereas U/Al values show a progressive drop from relatively high values (1.1) at the base of (and prior to) the sapropel, to typical background values at the upper boundary. The $\mathrm{Mo} / \mathrm{Al}$ peak at $78 \mathrm{~cm}$ may reflect Mo uptake during reventilation and cessation of the S1 event, possibly related to concomitant Fe and Mn precipitation.

\subsection{Fe speciation profiles for core 9509}

The speciation vs. depth profile for the five $\mathrm{Fe}_{\mathrm{HR}}$ fractions shows a number of significant differences between the sapropel and background sediments (Fig. 4a; Table 2). The $\mathrm{Fe}_{\mathrm{ox}}$ fraction (crystalline Fe (oxyhydr) oxides) is the most abundant fraction in the background sediments ( $\geq 1.2 \mathrm{wt} \%$ ), but shows a significant drop in the sapropel, down to a minimum values of $\sim 0.6 \mathrm{wt} \%$ in the lower sapropel at $152-162 \mathrm{~cm}$. The high background $\mathrm{Fe}_{\mathrm{ox}}$ content of these Nile Fan sediments is compatible with high Fe (oxyhydr)oxide contents in Nile River particulates (Poulton and Raiswell, 2002). The $\mathrm{Fe}_{\text {mag }}$ shows a drop from values of $0.3-0.4 \mathrm{wt} \%$ in the non-sapropel sediments to $\sim 0.17 \mathrm{wt} \%$ in the sapropel at $152-162 \mathrm{~cm}$. The $\mathrm{Fe}(\mathrm{II})_{\mathrm{HCl}}$ and $\mathrm{Fe}(\mathrm{III})_{\mathrm{HCl}}$ pools remain approximately constant across the sapropel, at $\sim 0.02-0.04 \mathrm{wt} \%$ and $\sim 0.30-0.37 \mathrm{wt} \%$, respectively. In contrast, $\mathrm{Fe}_{\mathrm{py}}$ rises from background values of $\leq 0.1 \mathrm{wt} \%$ in the non-sapropel sediment, to maxima of $0.45-0.62 \mathrm{wt} \%$ at $152-162 \mathrm{~cm}$, coincident with the minima observed in $\mathrm{Fe}_{\text {ox }}$ (Fig. 4a). Thus, there appears to be a reciprocal relationship whereby pyrite forms following the reductive dissolution of crystalline iron oxides and magnetite. Notably these reciprocal processes begin in the $5-10 \mathrm{~cm}$ sediment immediately below the sapropel, This zone has been termed the 'protosapropel' by some workers (e.g., Murat and Got, 1987).

A second feature of the Fe speciation data concerns the relatively high, and constant, concentrations of $\mathrm{Fe}(\mathrm{III})_{\mathrm{HCl}}$ (Fig. 4). The ferrihydrite that dominantly comprises this $\mathrm{Fe}$ pool is one of the most reactive $\mathrm{Fe}$ minerals towards dissolved sulphide (Poulton, 2003; Poulton et al., 2004). This fraction would not be expected to persist in sulphidic sediments, particularly when there is clear evidence of pyrite forming at the expense of more crystalline Fe (oxyhydr)oxides (Fig. 4; Canfield, 1989). This may, however, relate to oxidation of the sediments during storage. While oxidation has clearly not entirely altered the Fe mineralogy of these sediments, it appears likely that the relatively minor concentrations of $\mathrm{Fe}(\mathrm{III})_{\mathrm{HCl}}$ present throughout the depth profile may be a consequence of post-sampling oxidation of reduced Fe species, which could originally have comprised $\mathrm{Fe}_{\mathrm{Avs}}, \mathrm{Fe}_{\mathrm{py}}$, or possible $\mathrm{Fe}(\mathrm{II})_{\mathrm{HCl}}$. Given this possibility, we consider that our measured $\mathrm{Fe}_{\mathrm{py}}$ contents represent minimum original concentrations. Thus, to account for this, we also consider corrected (maximum) $\mathrm{Fe}_{\mathrm{py}}$ contents (Table 2), which are calculated as $\mathrm{Fe}_{\mathrm{py}}+\mathrm{Fe}(\mathrm{III})_{\mathrm{HCl}}$, and which assumes that all $\mathrm{Fe}(\mathrm{III})_{\mathrm{HCl}}$ originated from $\mathrm{Fe}$ sulphide oxidation. In support of this approach, we note that the drop observed in $\mathrm{Fe}_{\mathrm{ox}}+\mathrm{Fe}_{\text {mag }}$ between the upper boundary of the sapropel and the minimum observed for these fractions at a depth of $162 \mathrm{~cm}(0.86 \mathrm{wt} \%$; Table 2) corresponds well to the concomitant increase in $\mathrm{Fe}_{\mathrm{py}}+\mathrm{Fe}(\mathrm{III})_{\mathrm{HCl}}$ observed over the same interval $(0.72 \mathrm{wt} \%)$, suggesting that the $\mathrm{Fe}(\mathrm{III})_{\mathrm{HCl}}$ pool likely originated from post-sampling oxidation of $\mathrm{Fe}$ sulphide minerals. We stress, however, that this analysis only alters absolute concentrations of $\mathrm{Fe}_{\mathrm{py}}$, and the overall depth trends remain robust.

\subsection{Fe and Mo isotope compositions}

The depth profile for Fe isotopes shows restricted variation, with $\delta^{56} \mathrm{Fe}$ values ranging between -0.05 and $+0.08 \%$ (Table 1; Fig. $4 \mathrm{~b}$ ). There are no major, consistent differences between the sapropel and background samples The range of values $(0.02 \pm 0.04 \%$; 1 sd $)$ falls close to the 'modern marine sediment' $\delta^{56} \mathrm{Fe}$ values of $0.13 \pm 0.07 \%$ originally measured by Beard et al. (2003a) (recalculated to the IRMM014 standard). Sediments of the oxic shelf of the Black Sea give a mean value of $0.20 \pm 0.02 \%$ (Severmann et al., 2008; recalculated to the IRMM-014 standard). Closer to the Nile Fan in terms of setting, the estuarine suspended load of the North River, Massachusetts also showed a relatively narrow range of $\delta^{56} \mathrm{Fe}$ values $(0.07 \pm 0.05 \%$ ) over a wide range of salinities and Fe/Al values (Escoube et al., 2009). 

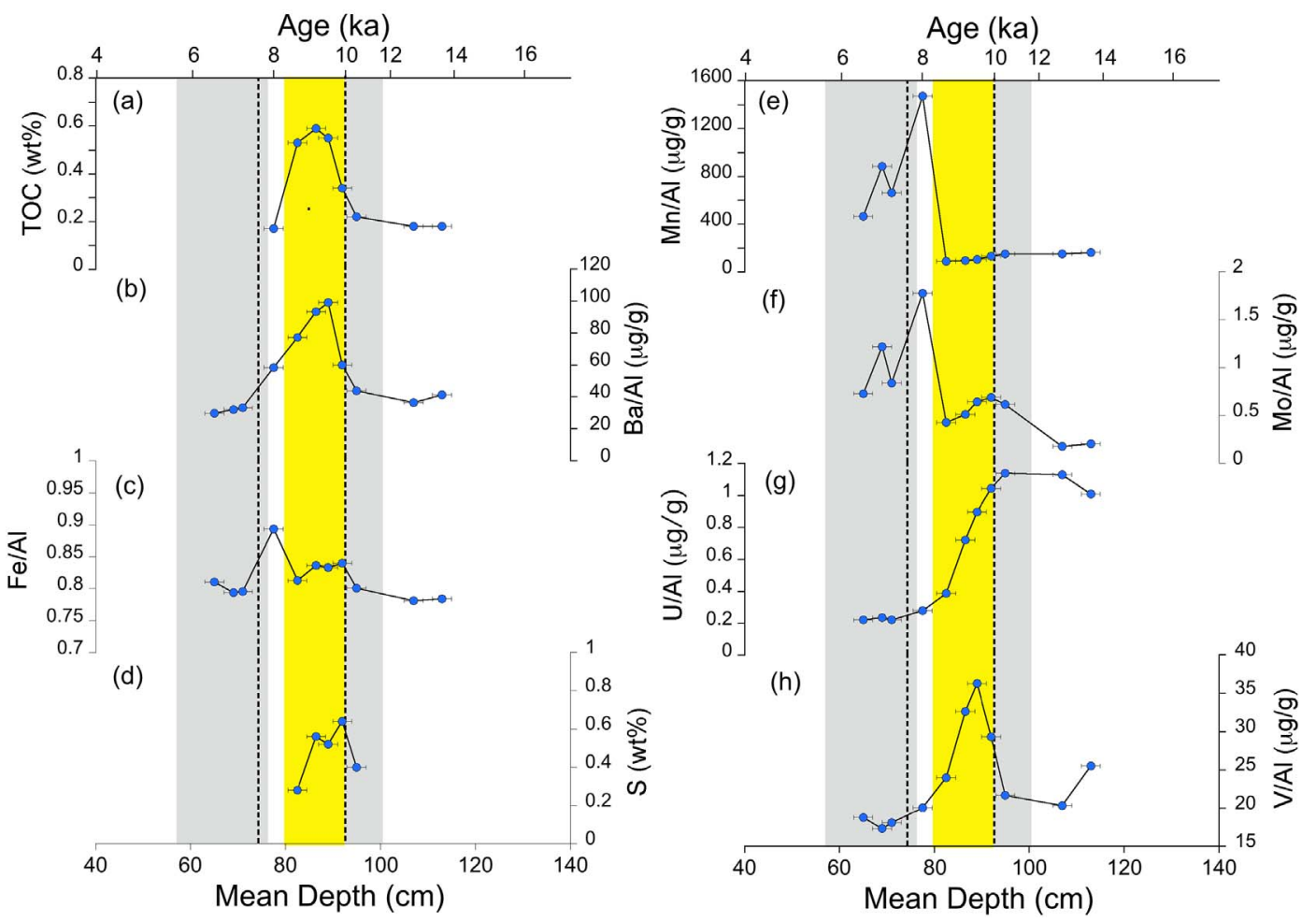

Fig. 3. Geochemical profiles of trace and major elements and TOC for 9501 bulk samples. Symbols, boundaries and shading as in Fig. 2.

In contrast to the restricted variation of Fe isotopes, the depth profile for Mo isotopes in the 9509 bulk samples is more complex (Table 1; Fig. 5a). The $\delta^{98 / 95}$ Mo values of -0.1 to $1.1 \%$ fall between those of anoxic (non-euxinic) continental margin marine sediments $(\sim 1.6 \%)$ and open-marine oxic sediments $(\sim-0.7 \% 0)$. The depth profile data can be subdivided into three segments:

1. Rise of $\delta^{98 / 95} \mathrm{Mo}$ in the pre-sapropel sediment from background levels of $\sim 0.2 \%$ at $260-280 \mathrm{~cm}(\sim 14 \mathrm{ka})$ to $0.7 \%$ at $178 \mathrm{~cm}$ $(\sim 10 \mathrm{ka}$; just below the sapropel lower boundary) and a corresponding decrease from high $\delta^{98 / 95}$ Mo values of $1.07 \%$ at the upper sapropel boundary to background values of $-0.07 \%$ in the most recent samples.

2. Asymmetric minimum $\delta^{98 / 95} \mathrm{Mo}$ values of $0.3 \%$ at $150 \mathrm{~cm}$ $(\sim 8.8 \mathrm{ka})$ and $0.55 \%$ at $126 \mathrm{~cm}(\sim 7.5 \mathrm{ka})$, separated by intermediate peak values of $\sim 0.65$ to $0.73 \%$ at $134-138 \mathrm{~cm}$. The location of these latter samples corresponds to the timing of the $8.2 \mathrm{ka}$ reventilation event evident in the TOC, $\mathrm{Ba} / \mathrm{Al}$ and element profiles (Fig. 2). The general double minimum form complements the double maximum profile found for RSTE (Fig. 2). A strongly defined asymmetric double minimum form $\left(\delta^{98 / 95} \mathrm{Mo}<-0.5 \%\right.$ ) separated by a reventilation maximum is a key feature of the sapropel S1 profile from ODP 967D (Azrieli-Tal et al., 2014).

3. A negative spike in $\delta^{98 / 95} \mathrm{Mo}$ (to $0.2 \%$ ) at the lower sapropel boundary. This negative spike possibly reflects the previously suggested mobilization of $\mathrm{Mn}$ in anoxic sediment developed during S1, which reprecipitated within oxic sediment beneath the organic-rich layer (Fig. 2f; Van Santvoort et al., 1996).

The $\delta^{98 / 95}$ Mo values of the $<63 \mu \mathrm{m}$ samples (5 sapropels and 1 overlying sediment) are, with the exception of the one sediment sample, mostly within error of equivalent bulk samples (Table 1; Fig. 5a). The $\delta^{98 / 95}$ Mo value $(0.6 \%)$ of the $<63 \mu$ m sediment sample immediately above the upper sapropel boundary is significantly lower than the value of $1.07 \%$ for the bulk sample, possibly suggesting that sieving preferentially removed isotopically heavy Mo from the bulk sediment.
Experimental measurements show that Mo adsorped onto minerals such as magnetite could be expected to be isotopically heavier than that concomitantly adsorped onto Fe (oxyhydr)oxides (Goldberg et al., 2009). Thus, preferential retention of detrital magnetite with higher $\delta^{98 / 95}$ Mo could potentially account for the isotopic difference. Despite the lower resolution of the $<63 \mu \mathrm{m}$ profile, its variation clearly matches the depletion trend observed in the bulk samples, particularly the minimum observed at $150 \mathrm{~cm}$.

The $\delta^{98 / 95}$ Mo values in core 9501 (Fig. 5b) show marked fluctuations in both the sapropel and pre-sapropel sediment: 1) a sharp drop from $1.2 \%$ in the lowermost sediment sample at $113 \mathrm{~cm}(\sim 13.5) \mathrm{ka}$ to $-0.9 \%$ at the lower sapropel boundary, and 2) a peak value of $0.95 \%$ in the sapropel at $86.5 \mathrm{~cm}$ ( $9 \mathrm{ka}$ ) (Fig. 5b), followed by a drop to background values of $\sim-0.1 \%$ at the sapropel termination.

\section{Discussion}

\subsection{Environmental conditions during sapropel development: elemental proxies}

Compared to deep water ( $>1800 \mathrm{mbsl}$ ) S1 sapropels (Emeis et al., 1998; de Lange et al., 2008; Gallego-Torres et al., 2010; Vance et al., 2004), both sapropels sampled in this study at depths of $<1000 \mathrm{mbsl}$ contain less organic matter (TOC $=0.5$ to $2 \mathrm{wt} \%$ ).

Characteristic trace and major element geochemical enrichments occur in both locations (Figs. 2, 3), but are shorter-lived and less well defined at site 9501 compared to 9509 . The comparable $\mathrm{Ba} / \mathrm{Al}$ $(\sim 6-9 \mu \mathrm{g} / \mathrm{g})$ and $\mathrm{RSTE}$ maximum values (V/Al, $\mathrm{Mo} / \mathrm{Al}, \mathrm{Sb} / \mathrm{Al}, \mathrm{U} / \mathrm{Al})$ in S1 between sites 9509 and MD04-2722 (Tachikawa et al., 2015) suggest similar redox conditions, despite the $900 \mathrm{~m}$ greater water depth of the latter. The enrichments in core 9509 thus suggest enhanced reducing conditions in the Nile Fan during sapropel S1 deposition. Similar Ba/Al and $\mathrm{V} / \mathrm{Al}$ ratios observed for sapropel S1 from core PS009PC, sampled close to 9509 at a water depth of 552 mbsl support this view (Hennekam et al., 2014).

The absence of a post-sapropel ventilation signal in core 9509 is 

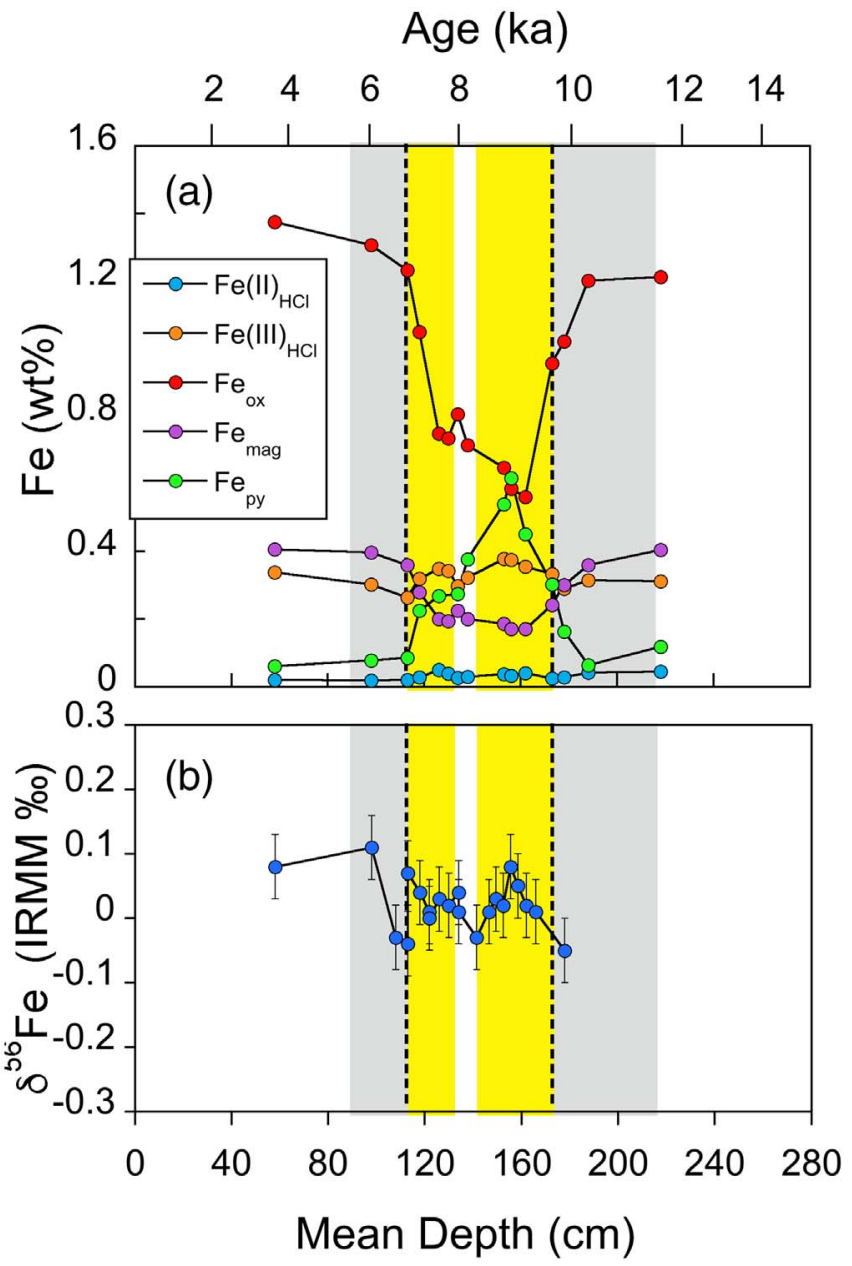

Fig. 4. (a) Fe speciation depth profile for highly reactive iron minerals $\left(\mathrm{Fe}_{\mathrm{HR}}\right)$. (b). Iron isotope depth profile of bulk extracts from core 9509 samples. Refer to text for an explanation of symbols. (For interpretation of the references to colour in this figure legend, the reader is referred to the web version of this article.)

consistent with sub-oxic conditions at the sapropel termination. Alternatively, if the rate of sedimentation was high enough, oxidative burndown may not have been fast enough to outpace sedimentation and conditions possibly could have been oxic. The presence of Mn and Fe peaks at the upper TOC boundary in 9501 indicate post-sapropel ventilation (Thomson et al., 1999). Whereas the 8.2 ka reventilation event and subsequent sapropel recovery is clearly shown in sapropel S1 from core 9509, the early collapse of S1 at site 9501 coincident with the 8.2 ka reventilation event is also evident (Figs. 2, 3). Taken together, the geochemical tracers indicate enhanced reducing conditions in the Nile Fan during sapropel S1 formation, as predicted by theoretic modeling (Grimm et al., 2015).

Cessation of deepwater renewal prior to S1 was a basin-wide phenomenon, in which global sea level rise following deglaciation was critical to the generation of oxygen-depleted water column conditions (Grimm et al., 2015; Rohling et al., 2015; Grant et al., 2016). The modest $\mathrm{Ba} / \mathrm{Al}, \mathrm{Mn} / \mathrm{Al}, \mathrm{U} / \mathrm{Al}$ and $\mathrm{V} / \mathrm{Al}$ enrichments observed in background sediments of core 9509 in the 16-14 ka period (Fig. 2) possibly suggest mild pre-S1 weakening of water column ventilation and increased biological productivity. High arboreal pollen levels (mainly evergreen oak), indicative of a warm wet climate beginning at $16 \mathrm{ka}$, were noted in core 9509 by Langgut et al. (2011), particularly during the Bolling-Allerod (14.6-12.6 ka) and S1 periods. Nevertheless, it should also be noted that there is no evidence from the RSTE proxies (Fig. 2) that the AHP onset resulted in marked deepening of reducing water column conditions. The onset of sapropel S1 at $173 \mathrm{~cm}(9.7 \mathrm{ka})$
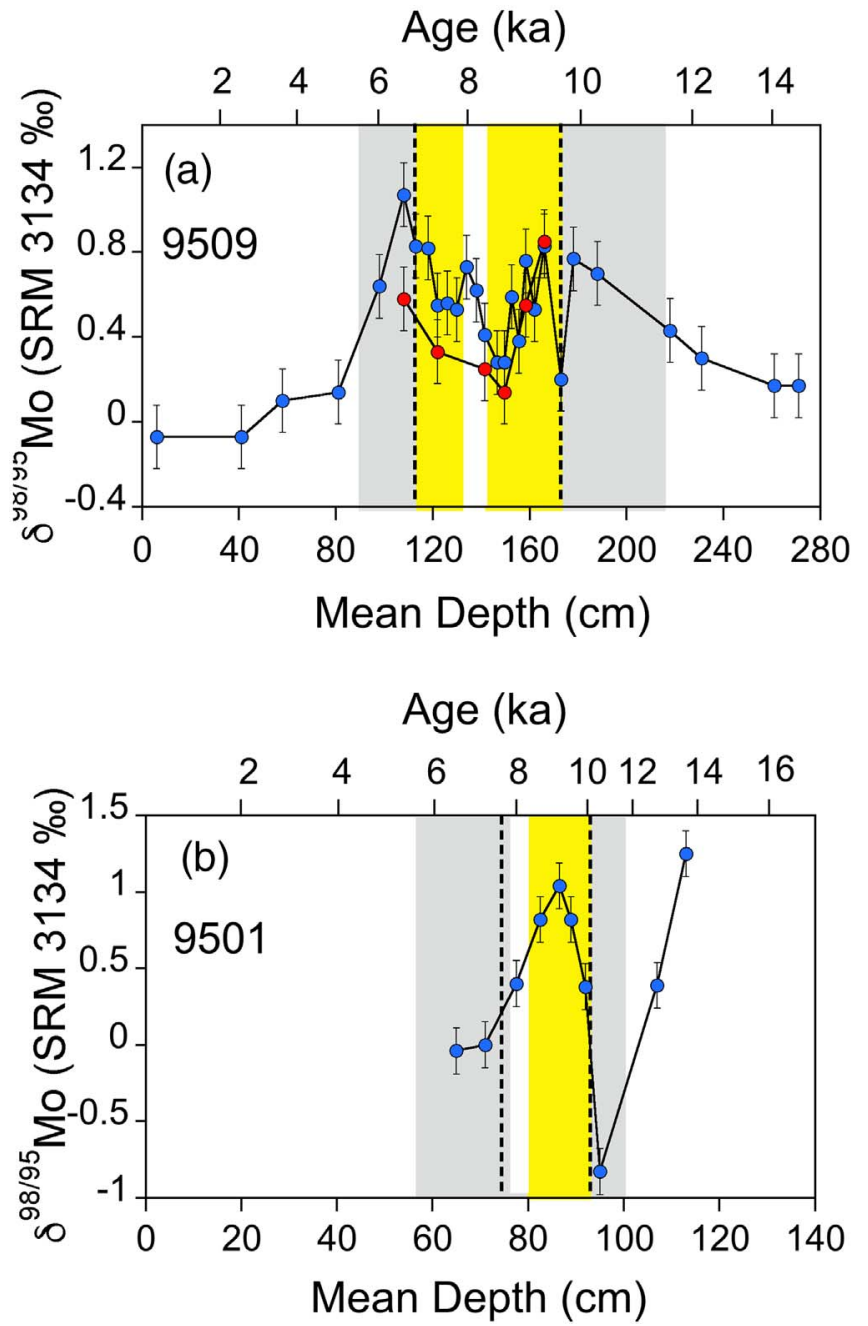

Fig. 5. (a) Molybdenum isotope profile for core 9509. Blue and red points represent bulk and $<63 \mu \mathrm{m}$ fraction samples, respectively. (b) Molybdenum isotope profile for core 9501 bulk samples. Colour shading and sapropel boundaries as given in Fig. 2. (For interpretation of the references to colour in this figure legend, the reader is referred to the web version of this article.)

was very sharp, with the most intense sapropel reducing conditions developing at $154-162 \mathrm{~cm}(\sim 8.9-9.2 \mathrm{ka})$. This early peak in the sapropel reducing conditions is a regional phenomenon of the Eastern Mediterranean Sea, also being observed in sapropel S1 in core 967D, where sulphidic bottom waters developed (Azrieli-Tal et al., 2014), core MD04-2722 (Fig. 3 of Tachikawa et al., 2015), and ODP cores 966, 969 and 964 (Gallego-Torres et al., 2010).

\subsubsection{Redox conditions indicated by $\mathrm{Mo}_{E F}-U_{E F}$ covariation}

Among the most successful trace element proxies for reduced oxygen water column conditions is Mo-U enrichment factor (EF) covariation (Algeo and Tribovillard, 2009), which utilizes the relative rates of authigenic $\mathrm{U}$ uptake during reduced-oxygen marine sedimentation compared to Mo uptake. Algeo and Tribovillard (2009) defined three diagnostic trends of $\mathrm{Mo}_{\mathrm{EF}}$ vs. $\mathrm{U}_{\mathrm{EF}}$ covariation (Fig. 6a): Particulate Shuttle (PS) in which Mo transfer to the sediment is accelerated through a particulate Mn-Fe-oxyhydroxide shuttle; Open Marine (OM) and restricted euxinic basin (BS). The $\mathrm{Mo}_{\mathrm{EF}} \mathrm{Vs}$. $\mathrm{U}_{\mathrm{EF}}$ covariations for $\mathrm{S} 1$ in core 9509 and its enclosing sediments are consistent with the OM trend (Fig. 6b). Sub-oxic bottom water conditions are indicated for the background sediments. These give way to more anoxic conditions in the sapropels, in which the rate of authigenic Mo accumulation increases relative to that of $\mathrm{U}$. However, bottom water conditions in which $\mathrm{Mo}_{\mathrm{EF}}$ 

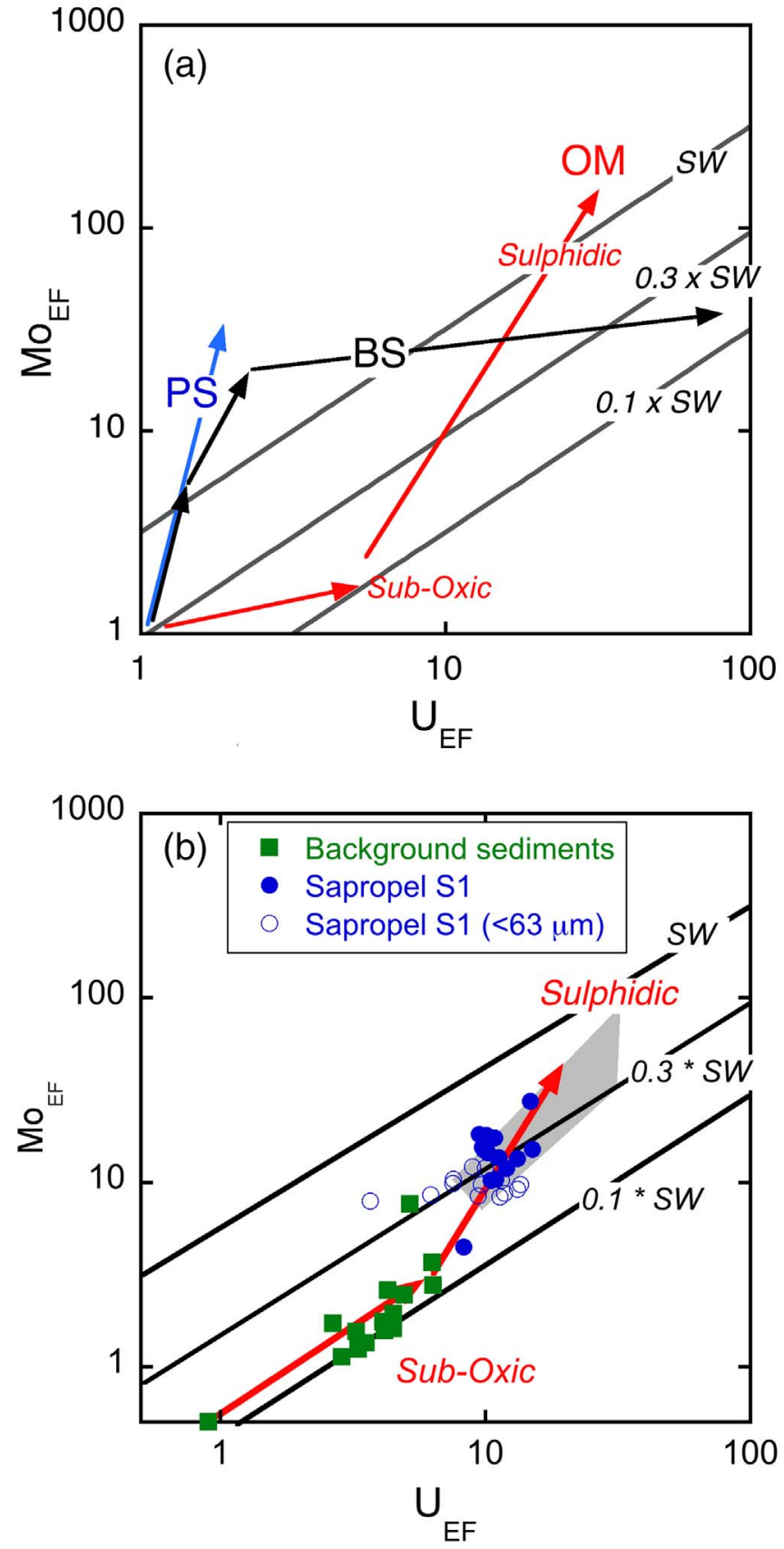

Fig. 6. $\mathrm{Mo}_{\mathrm{EF}}$ vs. $\mathrm{U}_{\mathrm{EF}}$ cross plots for 9509 samples. (a). Indicative trends of $\mathrm{Mo}_{\mathrm{EF}} \mathrm{vs}$. $\mathrm{U}_{\mathrm{EF}}$ defined by Algeo and Tribovillard (2009) for the Particulate Shuttle (PS), whereby Mo is preferentially shuttled to the sediment-water interface through Mn recycling, Open Marine systems (OM), and the Black Sea (BS) whereby initial rapid Mo uptake becomes retarded by Mo depletion in the highly restricted Black Sea Basin. The SW lines define trends along seawater $\mathrm{Mo}_{\mathrm{EF}}$ vs. $\mathrm{U}_{\mathrm{EF}}$ ratios $(\mathrm{SW}), 0.3 \times$ seawater ratios $(0.3 \times \mathrm{SW})$ and $0.1 \times$ seawater ratios $(0.1 \times \mathrm{SW})$, respectively. (b) $\mathrm{Mo}_{\mathrm{EF}}$ vs. $\mathrm{U}_{\mathrm{EF}}$ trends for core 9509 match the OM trend. Background sediments are consistent with sub-oxic bottom water conditions along the $0.1 \times$ SW line. The sapropel data mainly plot close to the $0.3 \times \mathrm{SW}$ ratios within the anoxic OM field, but do not reach seawater values suggested for sulphidic bottom water. The grey shaded area shows the stronger $\mathrm{Mo}_{\mathrm{EF}}$ and $\mathrm{U}_{\mathrm{EF}}$ enrichment trend exhibited by sapropel S1 at ODP site 967D (2550 mbsl) (Azrieli-Tal et al., 2014).

and $\mathrm{U}_{\mathrm{EF}}$ approach seawater ratios (SW line) and sulphidic conditions (indicated in Fig. 6b by the data trend for sapropel S1 at site ODP 967D) are not observed, suggesting that although anoxic bottom waters developed at peak sapropel conditions, they did not become sulphidic. The variable $\mathrm{U}$ and Mo data for 9501 do not fit any of the above EF trends (Table 1).

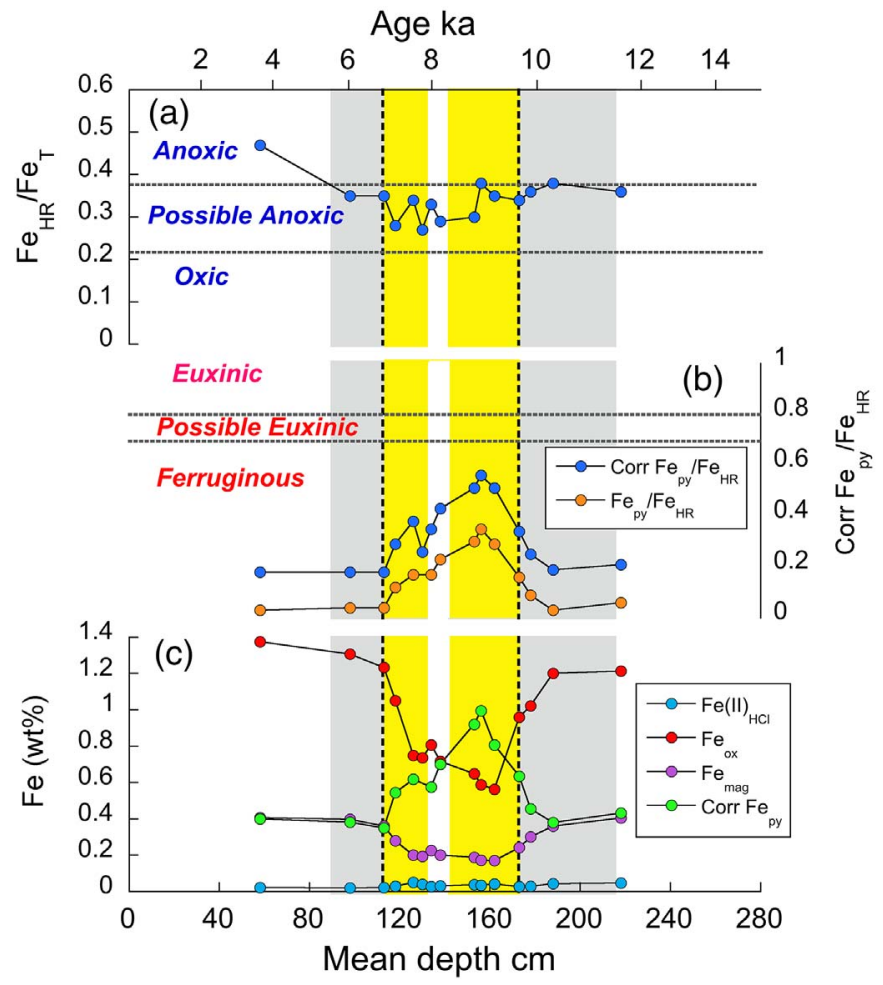

Fig. 7. Fe speciation profiles for sapropel S1 and background sediments from core 9509, (after Poulton and Canfield, 2005, 2011). (a) $\mathrm{Fe}_{\mathrm{HR}} / \mathrm{Fe}_{\mathrm{T}}$; (b) Corr $\mathrm{Fe}_{\mathrm{py}} / \mathrm{Fe}_{\mathrm{HR}}\left(\mathrm{Fe}_{\mathrm{py}} / \mathrm{Fe}_{\mathrm{HR}}\right.$ is shown for comparison); (c) mineralogical speciation in $\mathrm{Fe} w \mathrm{t} \%$ of the highly reactive iron minerals. (For interpretation of the references to colour in this figure legend, the reader is referred to the web version of this article.)

\subsection{Highly reactive Fe pools and Fe isotopes}

\subsubsection{Fe speciation constraints}

Elevated $\mathrm{Fe} / \mathrm{Al}$ total ratios greater than average crustal values of 0.55 to 0.6 are frequently associated with enrichments in reactive $\mathrm{Fe}$ during iron export from oxic shelves to deep euxinic basins (Lyons and Severmann, 2006; Lyons et al., 2009; Raiswell and Canfield, 2012). In the case of cores 9509 and 9501, Fe/Al values of the background sediments are unusually high $(\sim 0.8)$, reflecting high levels of Fe in the riverine source material (Poulton and Raiswell, 2002; Lyons and Severmann, 2006), rather than authigenic enrichment.

The $\mathrm{Fe}_{\mathrm{HR}} / \mathrm{Fe}_{\mathrm{T}}$ and $\mathrm{Fe}_{\mathrm{py}} / \mathrm{Fe}_{\mathrm{HR}}$ ratios derived from the $\mathrm{Fe}$ speciation studies on core 9509 (Table 2) are plotted as a function of sample depth in Fig. 7a and b. Using criteria summarized in Poulton and Canfield (2011) for 'normal' marine sediments, the $\mathrm{Fe}_{\mathrm{HR}} / \mathrm{Fe}_{\mathrm{T}}$ data plot in the 'possible anoxic' region during S1 formation, particularly during its early part and in the preceding AHP sediment (Fig. 7a). However, there is potential for the high $\mathrm{Fe}$ input from River Nile sediments to skew $\mathrm{Fe}_{\mathrm{HR}} / \mathrm{Fe}_{\mathrm{T}}$ ratios towards higher values (in the 'possible anoxic' region) if the sediment supplied to the region was relatively enriched in $\mathrm{Fe}$ (oxyhydr)oxides. Indeed, Poulton and Raiswell (2002) report relatively high $\mathrm{Fe}_{\mathrm{HR}} / \mathrm{Fe}_{\mathrm{T}}$ ratios of 0.26 for the River Nile, which is somewhat lower than most of the data for core 9509 (Fig. 7a), although it is difficult to directly compare a single analysis of modern River Nile sediment with the sediment deposited during $\mathrm{S} 1$ formation. Thus the $\mathrm{Fe}_{\mathrm{HR}}$ / $\mathrm{Fe}_{\mathrm{T}}$ data are equivocal, and building upon the trace metal systematics outlined above, could indicate anoxic non-sulphidic conditions with the operation of a benthic Fe shuttle (i.e., ferruginous water column conditions), or anoxic non-sulphidic conditions without the operation of a benthic Fe shuttle (i.e., non-ferruginous water column conditions). These possibilities are explored in further detail below.

The $\mathrm{Fe}_{\mathrm{py}} / \mathrm{Fe}_{\mathrm{HR}}$ profiles in Fig. $7 \mathrm{~b}$ are plotted for both the measured and corrected $\mathrm{Fe}_{\mathrm{py}}$ (Corr $\mathrm{Fe}_{\mathrm{py}} / \mathrm{Fe}_{\mathrm{HR}}$; see above) contents. Both plots 
show a significant rise in $\mathrm{Fe}_{\mathrm{py}} / \mathrm{Fe}_{\mathrm{HR}}$ from lowest values in the background sediment to peak values at $152-162 \mathrm{~cm}$ in the lower sapropel. It should be noted, however, that it is entirely possible that Corr $\mathrm{Fe}_{\mathrm{py}} /$ $\mathrm{Fe}_{\mathrm{HR}}$ values are over-estimated for the sediments deposited either side of the sapropel, since the $\mathrm{Fe}(\mathrm{III})_{\mathrm{HCl}}$ contents of these samples may have originated from oxidation of surface-reduced Fe(II), rather than pyrite or AVS. This would be consistent with the mass balance constraints relating to pyrite formation at the expense of Fe (oxyhydr)oxide minerals discussed above. Nevertheless, regardless of these uncertainties and the interpretation of $\mathrm{Fe}_{\mathrm{HR}} / \mathrm{Fe}_{\mathrm{T}}$ ratios, non-euxinic conditions are indicated, with pyrite forming during diagenesis rather than under euxinic conditions in the water column (consistent with the $\mathrm{Mo}_{\mathrm{EF}}$ vs. $\mathrm{U}_{\mathrm{EF}}$ plot; Figs. 6, 7).

Two additional points can be noted. 1) Maximum pyrite contents at 152-162 cm depth are compatible with RSTE maxima in the lower sapropel (Fig. 2), whereas a significant drop in pyrite content occurs during the 8.2 ka reventilation event, followed by a slight rise in the upper sapropel. Pyrite formation and RSTE uptake were therefore concurrent diagenetic processes. 2) The Fe (oxyhydr)oxide to pyrite transformation begins in the $5-10 \mathrm{~cm}$ of sediment underlying the sapropel. In keeping with the concept of a 'protosapropel' this transformation would indicate that the transition to more reducing conditions began prior to sapropel inception. Alternatively, Passier et al. (1996, 1997, 1999) proposed that 'synsapropel' diffusion of microbial [HS-] from the sapropel into underlying sediment in conditions of high sulphide production would lead to pyrite formation through sulphidization of Fe oxides below the sapropel.

\subsubsection{Fe isotope constraints on pyrite formation}

The $\mathrm{Fe} / \mathrm{Al}$ ratios and $\delta^{56} \mathrm{Fe}$ values are key parameters of the benthic iron shuttle model (Lyons and Severmann, 2006; Severmann et al., 2008). A diagnostic feature of this model is an inverse correlation between $\mathrm{Fe} / \mathrm{Al}$ and $\delta^{56} \mathrm{Fe}$, which has been recognized in modern euxinic basins such as the Black Sea and the Baltic Sea (Severmann et al., 2008; Fehr et al., 2010). This diagnostic feature was found in the lower part of sapropel S1 at ODP 967D (Azrieli-Tal et al., 2014) and most likely represents shuttling of reduced Fe from the Eastern Mediterranean continental shelf into the Hellenic trench and formation of isotopically light authigenic pyrite.

The $\delta^{56} \mathrm{Fe}$ data for core 9509 bulk samples show a flat trend on a $\delta^{56} \mathrm{Fe}$ vs. Fe/Al plot (Fig. 8). There is no correlation between $\delta^{56} \mathrm{Fe}$ and $\mathrm{Fe} / \mathrm{Al}$ ratios, nor pyrite contents. The $\delta^{56} \mathrm{Fe}$ vs. Fe/Al data thus give no evidence for a significant benthic Fe shuttle, consistent with absence of euxinia suggested above (compare the trends for the Black sea and sapropel S1 at ODP967). The Fe/Al variation in sapropel S1 from core 9509 is related here (Section 3.1.1; Section 3.3) to changes in the amounts of detrital igneous minerals, rather than to authigenic pyrite formation in the water column. Such variations in detrital iron-bearing minerals (iron-titanium oxides, magnetite, pyroxene, amphibole) would not impart any significant change to the iron isotope composition of the sapropel, since the $\delta^{56} \mathrm{Fe}$ values of igneous minerals, suspended river sediments and marine sediments are all around 0.1\%o (Beard et al., 2003a; Poitrasson et al., 2014).

The lack of evidence for authigenic pyrite formation in euxinic bottom waters is consistent with diagenetic pyrite formation following bacterial sulphate reduction within sulphidic pore waters (Section 4.2.1). The $\delta^{56} \mathrm{Fe}$ data also show that the biogenic pore water sulphide that formed pyrite did not impart a definable negative Fe isotope signature to the sapropel. This is particularly clear in the pyrite-rich lower part of the sapropel where Corr $\mathrm{Fe}_{\mathrm{py}} / \mathrm{Fe}_{\mathrm{T}}$ values reach 0.22 , but no corresponding depletion in ${ }^{56} \mathrm{Fe}$ is observed. Essentially the sapropel sediment retains the iron isotope signature of terrestrial source material. Such an absence of Fe isotope fractionation during anoxic sedimentary diagenesis is not self-evident. Severmann et al. (2010) found that continental shelf benthic iron in porewater and some sediment fractions is enriched in the lighter Fe isotope due to bacterial reduction

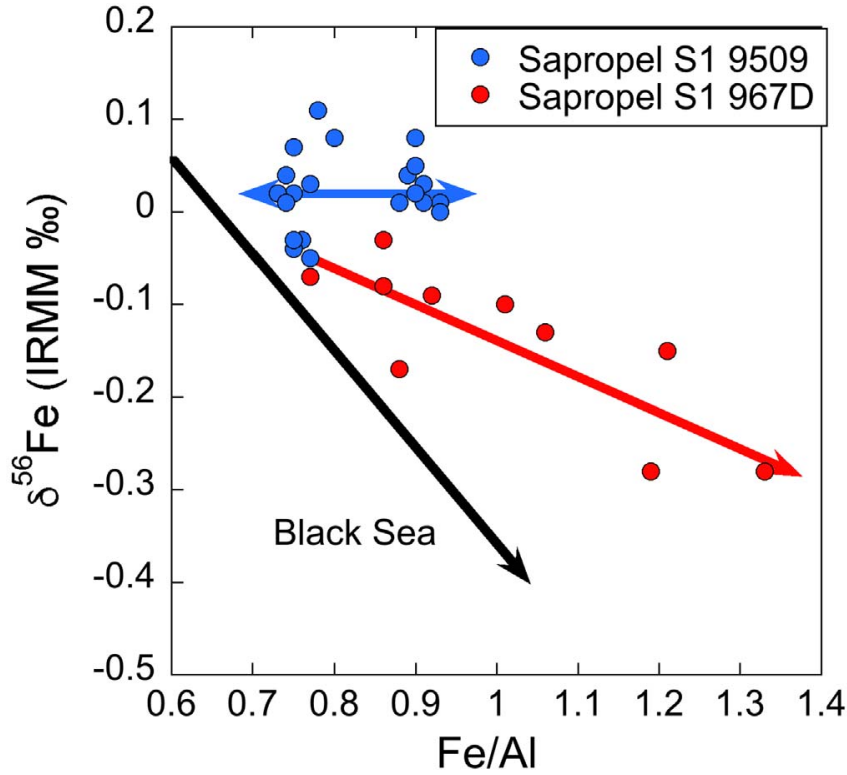

Fig. 8. Fe/Al vs. $\delta^{56} \mathrm{Fe}$ diagram for the data of this study. The lack of an inverse trend in the 9509 samples is contrasted with the data trend observed for samples from the lower half of deepwater sapropel S1 at site ODP 967D (Azrieli-Tal et al., 2014) and the steep trend observed for euxinic sediments of the Black Sea (Severmann et al., 2008). (For interpretation of the references to colour in this figure legend, the reader is referred to the web version of this article.)

of $\mathrm{Fe}(\mathrm{III})$ (oxyhydr)oxide minerals to $\mathrm{Fe}(\mathrm{II})_{\mathrm{aq}}$. Similarly, the positive kinetic iron isotope fractionation between $\mathrm{Fe}(\mathrm{II})_{\mathrm{aq}}$ and pyrite experimentally measured by Guilbaud et al. (2011) potentially allows for the formation of isotopically light Fe during sulphidization reactions. The absence of isotopically light $\mathrm{Fe}$ in the sapropel argues that the diagenetic reaction processes that formed pyrite occurred in a near-closed system with respect to Fe.

An important question arising from this data is whether $\mathrm{Fe} / \mathrm{Al}$ $\delta^{56} \mathrm{Fe}$ relations can be used to distinguish anoxic, ferruginous conditions in which a benthic Fe(II) shuttle is operational, from anoxic, nonsulphidic, non-ferruginous conditions in the water column. Scholz et al. (2014) and Chever et al. (2015) show that formation of isotopically heavy $\mathrm{Fe}$ (oxyhydr)oxides during $\mathrm{Fe}(\mathrm{II})$ shuttling in open marine oxygen minimum zones leads to a positive correlation between $\mathrm{Fe} / \mathrm{Al}$ and $\delta^{56} \mathrm{Fe}$. On the other hand, Staubwasser et al. (2013) show that kinetic fractionation during oxidative precipitation of $\mathrm{Fe}$ at the oxic-anoxic interface in the Baltic Sea leads to the formation of isotopically light $\mathrm{Fe}$ (oxyhydr)oxides and isotopically heavy $\mathrm{Fe}(\mathrm{II})$. Thus, the $\mathrm{Fe}$ mobilization in ferruginous waters potentially leads to opposing $\delta^{56} \mathrm{Fe}$ variations. The above examples are taken from modern oceanic settings. Nevertheless it can be noted that significant variability in the $\delta^{56} \mathrm{Fe}$ of pyrite has been suggested to be a diagnostic signal for ferruginous conditions in the ancient oceans (Tahata et al., 2015; Dauphas et al., 2017). Conversely, the absence of a $\delta^{56} \mathrm{Fe}$ shift from marine sedimentary values observed in this study could be diagnostic of anoxic, nonsulphidic, non-ferruginous conditions (i.e., without the operation of a benthic Fe shuttle) in the overlying water column. However, we stress that more detailed study of the isotope systematics of the benthic Fe shuttle under anoxic ferruginous conditions is required to substantiate this possibility, since positive fractionations generally imparted during partial oxidation of water column Fe(II) to Fe (oxyhydr)oxides could neutralize the light isotopic signature expected for water column Fe(II).

\subsection{Molybdenum isotope fractionation proxy for redox conditions}

The Mo isotope profile for core 9509 has two major redox segments (Fig. 5a). The first redox segment is that of the background sediments, 
which is marked by increase from lower $\delta^{98 / 95}$ Mo values in background sediments more distant from the sapropel to higher values close to the sapropel boundaries. Overall, this rise in $\delta^{98 / 95}$ Mo above lowest background values corresponds to the time of the AHP (grey shaded area in Fig. 5a). Sub-oxic conditions during background sediment formation are shown by the $\mathrm{Mo}_{\mathrm{EF}}$ vs. $\mathrm{U}_{\mathrm{EF}}$ plot (Fig. $6 \mathrm{~b}$ ), with the highest $\mathrm{Mo}_{\mathrm{EF}} / /$ $\mathrm{U}_{\mathrm{EF}}$ ratios occurring in the sediments immediate to the boundary (Table 1). There is no data to support substantial Mo uptake by Mn oxides since Mn concentrations in background sediments are typically low $(\sim 0.1 \mathrm{wt} \%)$. On the other hand, the Fe speciation data show that crystalline $\mathrm{Fe}$ oxides (mainly $\mathrm{Fe}_{\mathrm{ox}}$ ) dominate the $\mathrm{Fe}_{\mathrm{HR}}$ fraction in much of the background sediment (Fig. 7c). Thus, adsorption to Fe (oxyhydr) oxides is a likely mechanism for Mo uptake and isotope fractionation in these sediments, and is also consistent with non-sulphidic sub-oxic conditions (Goldberg et al., 2012). In the sediments at or near the lower and upper sapropel boundaries (Fig. 5a), Mo adsorption to pyrite might become relevant (Poulson-Brucker et al., 2012). Since, both adsorption to $\mathrm{Fe}$ (oxyhydroxides) and pyrite involve negative isotopic fractionations (with respect to the aqueous solution species), it might be that the observed rise in $\delta^{98 / 95}$ Mo in the underlying sediment reflects gradual increase in $\delta^{98 / 95}$ Mo of the porewater solution species as Mo is taken up onto the solid minerals by adsorption. Conversely, the drop in $\delta^{98 / 95} \mathrm{Mo}$ in the overlying sediment could reflect progressive release of Mo during desorption.

The main feature of the 9509 sapropel S1 is the asymmetric double minimum $\delta^{98 / 95}$ Mo form with the intermediate peak corresponding to the 8.2 ka ventilation event (Fig. 5a). The $\delta^{98 / 95}$ Mo values of the $<$ $63 \mu \mathrm{m}$ fraction also show the lower sapropel minimum value $(0.1 \% 0)$. However, the second peak is not evident in the $<63 \mu \mathrm{m}$ samples. It is clear that sampling was not made at sufficiently high spatial resolution to identify the second post ventilation event minimum. The $\delta^{98 / 95} \mathrm{Mo}$ minima in lower sapropel in both fractions broadly correspond to the highest intensity of the signals from $\mathrm{Ba} / \mathrm{Al}$, TOC and RSTE (Fig. 2) where reducing conditions would be at a maximum. As previously noted, the speciation plot for Fe (Fig. 7) shows that pyrite contents reach a maximum $(\sim 156 \mathrm{~cm})$ in the lower sapropel, at depths corresponding to the highest intensity of the geochemical proxies. Nevertheless, a slight lag is evident in the lower sapropel between the $\delta^{98 /}$ ${ }^{95} \mathrm{Mo}$ minimum at $\sim 150 \mathrm{~cm}$, compared to pyrite, $\mathrm{Ba} / \mathrm{Al}$ and RSTE maximum values.

The data profiles for the lower portion of the sapropel support a general connection between peak reducing conditions, pyrite formation and a decrease of $\delta^{98 / 95}$ Mo values. Consequently, the development of minimum values in 9509 sapropel can be examined using models based speciation controls during diagenetic bacterial sulphate reduction in anoxic porewater. During such reduction, $\mathrm{MoO}_{4}{ }^{2-}$ may sequentially form thiomolybdate species: $\mathrm{MoO}_{3} \mathrm{~S}^{2-}, \mathrm{MoO}_{2} \mathrm{~S}_{2}{ }^{2-}, \mathrm{MoOS}_{3}{ }^{2-}$ and $\mathrm{MoS}_{4}{ }^{2-}$. As noted in the introduction, non-quantitative Mo uptake as thiomolybdate species may lead to isotopic fractionation between molybdate and thiomolybdate species. Quantum mechanical calculations show that thiomolybdate species are negatively fractionated relative to $\mathrm{MoO}_{4}{ }^{2-}$ (seawater $\delta^{98 / 95} \mathrm{Mo}=2.3 \%$; Siebert et al., 2003) by 1.2 to 1.5\%o per S substitution (Tossell, 2005; B3LYP calculations). Fractionation factors of this magnitude have recently been experimentally confirmed using ion chromatographic thiomolybdate species separation and detection by MC-ICPMS (Kerl et al., 2017). Progressively sulphidic conditions in porewaters could thus lead to lower Mo isotopic compositions as the more sulphidized thiomolybdate and more isotopically fractionated species become dominant (Neubert et al., 2008; Nägler et al., 2011; Azrieli-Tal et al., 2014). Although previous studies specifically refer to syngenetic sapropel formation from euxinic bottom waters, they could equally apply to porewater sulphidic conditions during diagenesis (Wagner et al., 2017). Erickson and Helz (2000) also note that even in euxinic waters, Mo uptake commonly takes place by diagenetic pathways. The particulate uptake of thiomolybdate species by sedimentary sulphide minerals (e.g. pyrite) increases with the degree
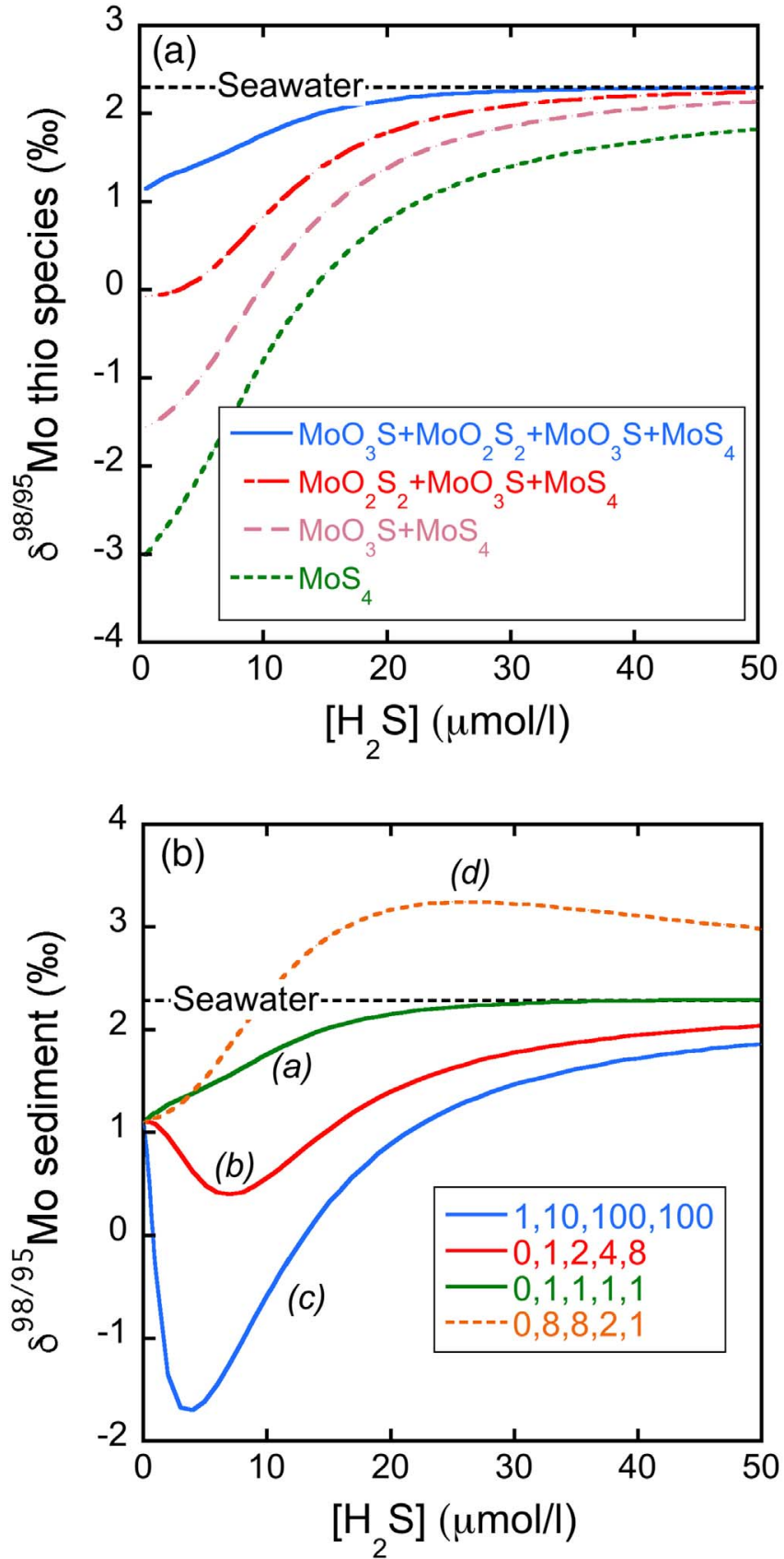

Fig. 9. (a) The Mo isotope composition of thiomolybdate solution species $\left(\delta^{98 / 95}\right.$ Mo (thio species)) vs. aqueous $\mathrm{H}_{2} \mathrm{~S}$ concentration. Four calculations are made for various combinations of thiomolybdate ions indicated in the figure legend. The molecular speciation of the thiomolybdate ions is according to their thermodynamic equilibrium proportions (data of Erickson and Helz, 2000). The bulk solution Mo isotopic composition is that of seawater $\left(\delta^{98 / 95} \mathrm{Mo}=2.3 \%\right.$; Siebert et al., 2003). The calculation of Mo isotope fractionation factors follows the method described by Nägler et al. (2011), using fractionation factors calculated using the B3LYP code (Tossell, 2005). Please note that this diagram corrects Fig. 8b of Azrieli-Tal et al. (2014) in which the isotopic composition of the thiomolybdate species was incorrectly plotted. The conclusions of Azrieli-Tal et al. (2014) regarding the development of negative $\delta^{98 / 95}$ Mo values through the preferential uptake of tri- and tetra-thiomolybdate species remain the same. (b) Variations of $\delta^{98 / 95}$ Mo sediment vs. aqueous $\mathrm{H}_{2} \mathrm{~S}$ concentration calculated using kinetic models for Mo particulate uptake as thiomolybdate species. Thiomolybdate species are calculated in their equilibrium thermodynamic proportions and seawater bulk $\delta^{98 / 95} \mathrm{Mo}$ is assumed as in (a), but rates of their uptake into the sediment are varied as indicated in the legend and text. (For interpretation of the references to colour in this figure legend, the reader is referred to the web version of this article.) 
of sulphidization (Helz et al., 1996; Erickson and Helz, 2000; Bostick et al., 2003; Helz et al., 2004; Dahl et al., 2010).

One model for the Mo isotope depletion is based on formation of thiomolybdate species according their thermodynamic equilibrium proportions (Neubert et al., 2008; Nägler et al., 2011; Azrieli-Tal et al., 2014). Calculations of the isotopic composition of various combinations of thiomolybdate species in equilibrium with $\mathrm{H}_{2} \mathrm{~S}$ (Fig. 9a) shows that preferential particulate affinity to the tetra- and tri-sulphide thiomolybdate species with increasing $\left[\mathrm{H}_{2} \mathrm{~S}\right]$ could lead to lower $\delta^{98 / 95} \mathrm{Mo}$ values, while increasing the uptake of RSTE.

A more detailed insight into Mo isotope depletion due to kinetically controlled preferential particulate uptake of Mo by the more sulphidized species is presented in Fig. 9b in a series of calculations of $\delta^{98 /}$ ${ }^{95} \mathrm{Mo}$ of the sulphidic sediment vs. $\left[\mathrm{H}_{2} \mathrm{~S}\right]$, where rates of particulate uptake of thiomolybdate species are varied. The calculation assumes that thiomolybdate species form in their equilibrium molecular proportions, but that rates of particulate scavenging of these species by the sediment are the rate controlling parameters. Three representative curves are plotted for the following scavenging rate conditions: a) all four thiomolybdate species are scavenged at the same rate, but molybdate remains unscavenged (curve $0,1,1,1,1$ ); $b$ ) the rates of thiomolybdate scavenging are doubled with $\mathrm{S}$ substitution (curve $0,1,2,4$, 8 ); c) rates increase by an order of magnitude with $S$ substitution (curve $0,1,10,100,1000)$. Note that this empirical kinetic model based on progressively differential rates of scavenging of the thiomolybdate species differs from the kinetic isotopic fractionation model of Dahl et al. (2010), where the rate of Mo removal (scavenging) is fast compared to the slow reaction kinetics of thiomolybdate formation.

It is evident that the lowest Mo isotope compositions occur when the rates of scavenging show a progressive increase with $\mathrm{S}$ substitution (curves b and c). $\delta^{98 / 95}$ Mo sediment values increase with $\left[\mathrm{H}_{2} \mathrm{~S}\right]$ when there is no preferential uptake (curve (a)). The doubling rate parameters for curve (b) $(0,1,2,4,8)$ most well fit the $\delta^{98 / 95}$ Mo depletions seen in the lower part of 9509 sapropel S1. Indeed two scenarios involving ${ }^{98 / 95}$ Mo depletion to a minimum with $\left[\mathrm{H}_{2} \mathrm{~S}\right]$ variation can be envisaged on the basis of curve (b): depletion to a minimum, followed by a rise in $\delta^{98 / 95}$ Mo with increasing $\left[\mathrm{H}_{2} \mathrm{~S}\right]$; depletion to a minimum with increasing $\left[\mathrm{H}_{2} \mathrm{~S}\right]$ followed by a rise in $\delta^{98 / 95} \mathrm{Mo}$ with declining $\left[\mathrm{H}_{2} \mathrm{~S}\right]$. Of these two scenarios, the latter is considered the more probable since other data such as $\mathrm{Ba} / \mathrm{Al}$, TOC and RSTE indicate that the $\delta^{98 / 95}$ Mo minima are associated with maximum reducing conditions, and therefore maximum porewater $\mathrm{H}_{2} \mathrm{~S}$. Following the recent XAFS spectroscopic study of Wagner et al. (2017) showing that intermediate thiomolybdate $\left(\mathrm{MoO}_{\mathrm{x}} \mathrm{S}_{4-\mathrm{x}}{ }^{2-}\right)$ species, Fig. 9b also plots a graph in which the intermediate thiomolybdate species dominate Mo uptake rates (hashed line curve d; $0,4,8,2,1$ ). Low $\delta^{98 / 95}$ Mo values are generated, but the values show an increase with increasing $\left[\mathrm{H}_{2} \mathrm{~S}\right]$ that are not consistent maximum reducing conditions.

The coincidence of pyrite growth and Fe oxide depletion with RTSE maxima suggests that pyrite could be the dominant mineral species for thiomolybdate particulate uptake. The approximately $6 \mathrm{~cm}$ slight lag in the lower sapropel between the $\delta^{98 / 95}$ Mo minimum and pyrite maximum values in the lower sapropel could reflect an offset due to the diffusional rate controlled kinetic uptake of Mo by pyrite during diagenesis. This $6 \mathrm{~cm}$ of sediment represents $\sim 260 \mathrm{yr}$ of sediment deposition (using the dating of Almogi-Labin et al., 2009). Using the 1-dimensional mean diffusion distance equation: $x^{2}=2 D t(x=$ distance, $\mathrm{D}=$ diffusion coefficient), the $\mathrm{D}$ value corresponding to a time interval of $260 \mathrm{yr}$ is $\sim 2 * 10^{-9} \mathrm{~cm}^{2} / \mathrm{s}$. This apparent diffusion coefficient is more than four orders of magnitude faster than the free ion diffusion rate, implying either very high sediment tortuosity and/or significant lag in Mo isotope uptake relative to pyrite formation in the high sedimentation rate environment.

All the above calculations assume that seawater $\delta^{98 / 95}$ Mo $=2.3 \%$. This value could be different in the Nile plume due to the influx of river Nile dissolved Mo. The average present-day main Nile $\delta^{98 / 95}$ Mo is about
$0.3 \%$, and dissolved [Mo] during the wet monsoonal season is $\sim 8 \mathrm{nmol}$ (Archer and Vance, 2008). Simple mass calculation for equivolume mixing of this Nile input with seawater $([\mathrm{Mo}]=105 \mathrm{nmol})$ would give a mean $\delta^{98 / 95} \mathrm{Mo}=2.04 \%$. This would not significantly alter the Mo isotopic values calculated in Fig. 9.

The core 9501 profile is characterized by large $\delta^{98 / 95}$ Mo shifts; from values of $>1 \%$ to $-1 \%$ in the pre-sapropel sediment, a rise to a maximum of $1 \%$ within the sapropel, followed by a rapid decrease to values of $\sim 0 \%$ at about $8.2 \mathrm{ka}$ (Fig. $5 \mathrm{~b}$ ), most obviously associated with the reventilation event. The $\delta^{98 / 95}$ Mo profile of core 9501 significantly differs from that of core 9509 and cannot be modeled using the Fe-oxide adsorption/kinetic thiomolybdate uptake mechanisms proposed for core 9509. Nor do the data trends provide direct support for the alternative model of Reitz et al. (2007) in which an initial light oxic $\left(\mathrm{MnO}_{\mathrm{x}}\right.$ adsorption) Mo isotope signal in the sapropel is transported downward to a more reduced sub-oxic zone during burial diagenesis. The marked $\delta^{98 / 95}$ Mo variations in the pre-sapropel sediment may reflect water column redox fluctuations in the open sea at $\sim 1000 \mathrm{~m}$ depth. These variations are not found in pre-sapropel sediment of the Nile Fan at site 9509 where the Nile suspended matter outflow may be buffering the redox state close to the Fe (oxyhydr)oxide-Fe(II) $)_{\mathrm{aq}}$ couple.

\section{Conclusions}

Anoxic, non-sulphidic, bottom water conditions occurred over the course of sapropel S1 deposition at intermediate water depths in the Nile Fan, with sub-oxic water conditions prevailing in the immediately preceding and post-dating sediments. Redox conditions were more reducing in the early phase of sapropel formation. The chemical element proxy signals for the 9509 sapropel are similar in magnitude to those of sapropel S1 recovered in core MD04-2722 on the continental slope at $1780 \mathrm{mbsl}$ (Tachikawa et al., 2015), but stronger than those of sapropel S1 from core 9501 recovered at a similar depth to 9509 , but distal to the Nile Fan. The sapropel S1 reducing conditions in core 9509 were sufficiently robust that the sapropel recovered after the well known $8.2 \mathrm{ka}$ sapropel ventilation event, as recorded in several different proxies (Ba/ $\mathrm{Al}$, TOC, RSTE, $\delta^{98 / 95} \mathrm{Mo}$ ). The intermediate water depth interval of the Eastern Mediterranean Sea in the Nile Fan area thus developed an expanded oxygen minimum zone characterized by water column anoxia. The rapid development of anoxia at shallower conditions than the 1800 mbsl depth at which anoxic conditions occurred in the deep Eastern Mediterranean Sea basin has potential implications for the rate of expansion of anoxia in major river coastal fans, past and present.

Lowered water oxygenation conditions in core 9509 sediments preceding the sapropel $(\sim 14-16 \mathrm{ka})$ are suggested by $\mathrm{Ba} / \mathrm{Al}$ values and some of the metallic element data, consistent with results from previous studies (Kudnt et al., 2008; Schmiedl et al., 2010; Box et al., 2011; Tachikawa et al., 2015). However, there is no evidence for a shift to more reducing conditions at the beginning of the African Humid Period. Rather, the sharp enhancement in reducing conditions is synchronous with sapropel formation. Thus, evidence for a long period of water body stagnation preceding the SI event at intermediate water depths is ambiguous, though this finding does not necessarily impact on models showing that deep water stagnation preceded sapropel deposition (Grimm et al., 2015).

A major aim of this study was to identify the diagenetic and water column influences on the sedimentary record during sapropel deposition. Iron speciation indicates a major diagenetic reaction during sapropel formation in which pyrite grows at the expense of Fe (oxyhydr) oxides, the dominant highly reactive minerals in the pre-sapropel sediment. The pyrite growth occurs during bacterial sulphate reduction in anoxic porewaters below an anoxic, non-sulphidic water column, and reaches a maximum at peak reducing conditions. During these processes, the preservation in the sapropel of the Fe isotopic composition of marine sediments and the absence of a shift in $\delta^{56} \mathrm{Fe}$ to light values during periods of pyrite growth in the sapropel implies that diagenetic 
mobilization of iron in porewaters as $\mathrm{Fe}(\mathrm{II})_{\mathrm{aq}}$ and pyrite precipitation occurred in a closed system with respect to Fe. Both the Fe speciation data and the $\delta^{56} \mathrm{Fe}$ values point to the absence of a benthic Fe shuttle, i.e. an anoxic non-sulphidic-non-ferruginous water column. The Mo isotope compositions of sapropel S1 identify major redox periods whereby isotopic fractionation in the Mo isotope system switches from/ to dominantly sub-oxic Mo adsorption to Fe oxides and pyrite in the pre and post-sapropel sediments, to thiomolybdate species uptake in the sapropel. Peak sapropel anoxic reducing conditions are characterized by $\delta^{98 / 95} \mathrm{Mo}$ minima either side of the $8.2 \mathrm{ka}$ ventilation event. Modeling of Mo isotope fractionation during particulate uptake of the different thiomolybdate species indicates that $\delta^{98 / 95}$ Mo minima can be generated by modest increases in the rate of uptake of the more sulphidized thiomolybdate species as a function of $\mathrm{H}_{2} \mathrm{~S}$ concentration.

The diagnostic aspect of the Nile Fan Fe and Mo isotope data are evident when compared to Fe and Mo isotope data for sapropel S1 at ODP site 967D (2550 mbsl; weakly sulphidic bottom water; Azrieli-Tal et al., 2014) and the euxinic sediments of the Black sea (Arnold et al., 2004; Neubert et al., 2008: Nägler et al., 2011). Whereas, Fe isotopes show a progressive decrease to lower $\delta^{56} \mathrm{Fe}$ values with increasing sulphide (Fig. 7), $\delta^{98 / 95}$ Mo values show a shift from moderate to strong depletion ( $\left(\delta^{98 / 95}\right.$ Mo minima) in the weakly sulphidic bottom water at ODP-967D, but a return to sea-water values in the highly sulphidic euxinic conditions of the Black Sea. These differences demonstrate that the Fe and Mo isotope systematics provide a powerful diagnostic tool for exploring organic carbon rich sedimentary deposition in past and recent settings, especially when combined with Fe speciation studies.

Supplementary data to this article can be found online at https:// doi.org/10.1016/j.chemgeo.2017.10.028.

\section{Acknowledgements}

This research was supported by THE ISRAEL SCIENCE FOUNDATION GRANT 1140/12 to A.M. and M.B.M. and Swiss Science Foundation grant 200021_143262 to D.V. SWP acknowledges support from a Royal Society Wolfson Research Merit Award. We would like to thank Dr. Olga Yoffe of the GSI for help with the geochemical analyses of trace elements, Dr. Faina Gelman also of the GSI for TOC analyses, and to Ophir Tirosh from the Hebrew University for assisting with the clean laboratory work. Perceptive and very helpful critical comments of the reviewers and editor are very much appreciated by the authors.

\section{References}

Adkins, J., Demenocal, P., Eshel, G., 2006. The "African humid period" and the record of marine upwelling from excess 230Th in Ocean Drilling Program Hole 658C Paleoceanography 21 (4).

Algeo, T.J., Tribovillard, N., 2009. Environmental analysis of paleoceanographic systems based on molybdenum-uranium covaration. Chem. Geol. 268, 211-225.

Alley, R.B., Mayewski, P.A., Sowers, T., Stuiver, M., Taylor, K.G., Clark, U., 1997. Holocene climatic instability, a prominent, widespread event 8200 yr ago. Geology 25, 483-486.

Almogi-Labin, A., Bar-Matthews, M., Shriki, D., Kolosovsky, E., Paterne, M., Schilman, B., Ayalon, A., Aizenshtat, Z., Matthews, A., 2009. Climate variability during the last $\sim 90 \mathrm{kyr}$ in the southern and northern Levantine Basin as evident from the marine and speleothem records. Quat. Sci. Rev. 28, 2882-2896.

Archer, C., Vance, D., 2004. Mass discrimination correction in multiple-collector plasma source mass spectrometry: an example using $\mathrm{Cu}$ and $\mathrm{Zn}$ isotopes. J. Anal. At. Spectrom. 19, 656-665.

Archer, C., Vance, D., 2008. The isotopic signature of the global riverine molybdenum flux and anoxia in the ancient oceans. Nat. Geosci. 1, 597-600.

Arnold, G.L., Anbar, A.D., Barling, J., Lyons, T., 2004. Molybdenum isotope evidence for widespread anoxia in Mid-Proterozoic oceans. Science 304, 87-90.

Avnaim-Katav, S., 2005. Foraminifera as a Tool in Reconstructing Climate Variations During the Holocene in the Eastern Mediterranean Sea With an Affiliation to Historical and Archeological Records. M.A. thesis. Department of Maritime Civilizations, University of Haifa (English summary: maritime2.haifa.ac.il/eng/nl/ Rims32/33.pdf).

Azrieli-Tal, I., Matthews, A., Bar-Matthews, M., Almogi-Labin, A., Vance, D., Archer, C. Teutsch, N., 2014. Evidence from molybdenum and iron isotopes and molybdenum-uranium covariation for sulphidic bottom waters during Eastern Mediterranean Sapropel S1 formation. Earth Planet. Sci. Lett. 393, 231-242.
Barling, J., Anbar, A.D., 2004. Molybdenum isotope fractionation during adsorption by manganese oxides. Earth Planet. Sci. Lett. 217, 235.

Barling, J., Arnold, G.L., Anbar, A.D., 2001. Natural mass-dependent variations in the isotopic composition of molybdenum. Earth Planet. Sci. Lett. 193, 447-457.

Bar-Matthews, M., 2014. History of water in the Middle East and North Africa. In: Holland, H.D., Turekian, K.K. (Eds.), Treatise on Geochemistry, second edition. 14. Elsevier, Oxford, pp. 109-128.

Bar-Matthews, M., Ayalon, A., Kaufman, A., 2000. Timing and hydrological conditions of Sapropel events in the Eastern Mediterranean, as evident from speleothems, Soreq Cave, Israel. Chem. Geol. 169, 145-156.

Bar-Matthews, M., Ayalon, A., Gilmour, M., Matthews, A., Hawkesworth, C.J., 2003. Sealand oxygen isotopic relationships from planktonic foraminifera and speleothems in the eastern Mediterranean region and their implication for paleorainfall during interglacial intervals. Geochim. Cosmochim. Acta 67, 3181-3199.

Beard, B.J., Johnson, C.M., Von Damm, K., Poulson, R.L., 2003a. Iron isotope constraints on Fe cycling and mass balance in oxygenated Earth oceans. Geology 31, 629-632.

Beard, B.L., Johnson, C.M., Skulan, J.L., Nealson, K.H., Cox, L., Sun, H., 2003b. Application of $\mathrm{Fe}$ isotopes to tracing the geochemical and biological cycling of $\mathrm{Fe}$. Chem. Geol. 195, 87-117.

Bermin, J., Vance, D., Archer, C., Statham, P.J., 2006. The determination of the isotopic composition of $\mathrm{Cu}$ and $\mathrm{Zn}$ in seawater. Chem. Geol. 226, 280-297.

Bostick, B.C., Fendorf, S., Helz, G.R., 2003. Differential adsorption of molybdate and tetrathiomolybdate on pyrite $\left(\mathrm{FeS}_{2}\right)$. Environ. Sci. Technol. 37, 285-291.

Box, M.R., Krom, M.D., Cliff, R.A., Bar-Matthews, M., Almogi-Labin, A., Ayalon, A., Paterne, M., 2011. Response of the Nile and its catchment to millennial-scale climatic change since the LGM from Sr isotopes and major elements of East Mediterranean sediments. Quat. Sci. Rev. 30, 431-442.

Brumsack, H.-J., 2006. The trace metal content of recent organic carbon-rich sediments: implications for Cretaceous black shale formation. Palaeogeogr. Palaeoclimatol. Palaeoecol. 232, 344-361.

Butler, I.B., Archer, C., Vance, D., Olroyd, A., Rickard, D.T., 2005. Fe isotope fractionation on FeS formation in ambient aqueous solution. Earth Planet. Sci. Lett. 236, 430-442.

Calvert, S.E., Fontugne, M.R., 2001. On the late Pleistocene-Holocene sapropel record of climatic and oceanographic variability in the Eastern Mediterranean. Paleoceanography 16, 78-94.

Canfield, D.E., 1989. Reactive iron in marine sediments. Geochim. Cosmochim. Acta 53, 619-632.

Canfield, D.E., Raiswell, R., Westrich, J.T., Berner, R.A., 1986. The use of chromium re duction in the analysis of reduced inorganic sulfur in sediments and shales. Chem. Geol. 54, 149-155.

Canfield, D.E., Raiswell, R., Bottrell, S.H., 1992. The reactivity of sedimentary iron minerals toward sulphide. Am. J. Sci. 292, 659-683.

Canfield, D.E., Lyons, T.W., Raiswell, R., 1996. A model for iron deposition to euxinic Black Sea sediments. Am. J. Sci. 296, 818-834.

Casford, J.S.L., Abu-Zied, R., Rohling, E.J., Cooke, S., Boessenkool, K.P., Brinkhuis, H., De Vries, C., Wefer, G., Geraga, M., Papatheodorou, G., Croudace, I., Thomson, J., Wells, N.C., Lykousis, V., 2001. Mediterranean climate variability during the Holocene. Mediterr. Mar. Sci. 2, 45-55.

Chever, F., Rouxel, O.J., Croot, P.I., Ponzavera, E., Wuttig, K., Auro, M., 2015. Total dissolvable and dissolved iron isotopes in the water column of the Peru upwelling regime. Geochim. Cosmochim. Acta 162, 66-82.

Clarkson, M.O., Wood, R.A., Poulton, S.W., Richoz, S., Newton, R.J., 2016. Dynamic anoxic feruginous conditions during the end-Permian mass extinction and recovery. Nat. Commun. 7.

Craddock, P.R., Dauphas, N., 2011. Iron isotopic compositions of geological reference materials and chrondites. Geostand. Geoanal. Res. 35, 101-123.

Crosby, H.A., Roden, E.E., Johnson, C.M., Beard, B.L., 2007. The mechanisms of iron isotope fractionation produced during dissimilatory Fe(III) reduction by Shewanella putrefaciens and Geobacter sulfurreducens. Geobiology 5, 169-189.

Dahl, T.W., Anbar, A.D., Gordon, G.W., Rosing, M.T., Frei, R., Canfield, D.E., 2010. The behaviour of molybdenum and its isotopes across the chemocline and in the sediments of sulfidic Lake Cadagno, Switzerland. Geochim. Cosmochim. Acta 74, 144-163.

Dauphas, N., John, S.G., Rouxel, O., 2017. Iron isotope systematics. In: Teng, F.-Z., Watkins, J.M., Dauphas, N. (Eds.), Non-traditional Stable Isotopes. Rev. Mineral. Geochem. 82. pp. 415-510.

Emeis, K.-C., Schulze, H.-M., Struck, U., Sakamoto, T., Doose, H., Erlenkeuser, H., Howell, M., Kroon, D., Paterne, M., 1998. In: Robertson, A.H.F., Emeis, K.-C., Richter, C., Camerlenghi, A. (Eds.), Stable Isotope and Temperature Records of Sapropels From ODP Sites 964 and 967: Constraining the Physical Environment of Sapropel Formation in the Eastern Mediterranean Sea. 160. Ocean Drilling Program, College Station, TX, pp. 309-331 Proc. ODP Sci. Res.

Emeis, K.-C., Sakamoto, T., Wehausen, R., Brumsack, H.J., 2000. The sapropel record of the eastern Mediterranean Sea - results of Ocean drilling Program Leg 160 Paleogeogr. Paleoclimatol. Paleoecol. 158, 371-375.

Emeis, K.C., Schulz, H., Struck, U., Rossignol-Strick, M., Erlenkeuser, H., Howell, M.W., Kroon, D., Mackensen, A., Ishizuka, S., Oba, T., Sakamoto, T., Koizumi, I., 2003. Eastern Mediterranean surface water temperatures and $\delta^{18} \mathrm{O}$ composition during deposition of sapropels in the late Quaternary. Paleoceanography 18, 1005. http:// dx.doi.org/10.1029/2000PA000617.

Erickson, B.E., Helz, G.R., 2000. Molybdenum(VI) speciation in sulfidic waters: stability and lability of thiomolybdates. Geochim. Cosmochim. Acta 64, 1149-1158.

Escoube, R., Rouxel, O.J., Sholkovitz, E., Donard, O.F.X., 2009. Iron isotope systematics in estuaries: the case of North River, Massachusetts (USA). Geochim. Cosmochim. Acta 73, 4045-4059.

Fehr, M.A., Andersson, P.S., Hålenius, U., Mörth, C.-M., 2008. Iron isotope variations in 
Holocene sediments of the Gotland Deep. Geochim. Cosmochim. Acta 72, 807-826.

Fehr, M.A., Andersson, P.S., Halenius, U., Gustafsson, O., Morth, C.-M., 2010. Iron enrichments and Fe isotopic compositions of surface sediments from the Gotland Deep, Baltic Sea. Chem. Geol. 277, 310-322.

Filippidi, A., Triantaphyllou, M.V., de Lange, G.J., 2016. Eastern-Mediterranean ventilation variability during sapropel S1 formation, evaluated at two sites influenced by deep-water formation from Adriatic and Aegean Seas. Quat. Sci. Rev. 144, 95-106.

Gallego-Torres, D., Martinez-Ruiz, F., de Lange, G.J., Jimenez-Espejo, F.J., OrtegaHuertas, M., 2010. Trace-elemental derived paleoceanographic and paleoclimatic conditions for Pleistocene Eastern Mediterranean sapropels. Palaeogeogr. Palaeoclimatol. Palaeoecol. 293, 76-89.

Goldberg, T., Archer, C., Vance, D., Poulton, S.W., 2009. Mo isotope fractionation during adsorption to Fe (oxyhydr)oxides. Geochim. Cosmochim. Acta 73, 6502-6516.

Goldberg, T., Archer, C., Vance, D., Thamdrup, B., McAnena, A., Poulton, S.W., 2012. Controls on Mo isotope fractionations in a Mn-rich anoxic marine sediment, Gullmar Fjord, Sweden. Chem. Geol. 296, 73-82.

Grant, K.M., Grimm, R., Mikolajewicz, U., Marino, G., Ziegler, M., Rohling, E.J., 2016. The timing of Mediterranean sapropel deposition relative to insolation, sea-level and African monsoon changes. Quat. Sci. Rev. 140, 125-141.

Grimm, R., Maier-Reimer, E., Miklolajewicz, U., Schmiedl, G., Muller-Navarra, K., Adloff, F., Grant, K.M., Ziegler, M., Lourens, L.J., Emeis, K.-C., 2015. Late glacial initiation of Holocene eastern Mediterranean sapropel formation. Nat. Commun. http://dx.doi. org/10.1038/ncomms8099.

Guilbaud, R., Butler, I.B., Ellam, R.M., 2011. Abiotic pyrite formation a large Fe isotope fractionation. Science 332, 1548-1551.

Helz, G.R., Miller, C.V., Charnock, J.M., Mosselmans, J.F.W., Patrick, R.A.D., Garner, C.D., Vaughn, D.J., 1996. Mechanism of molybdenum removal from the sea and its concentration in black shales: EXAFS evidence. Geochim. Cosmochim. Acta 60, 3631-3642.

Helz, G.R., Vorlicek, T.P., Kahn, M.D., 2004. Molybdenum scavenging by iron monosulfide. Environ. Sci. Technol. 38, 4263-4268.

Hennekam, R., Jilbert, T., Schnetger, B., de Lange, G.J., 2014. Solar forcing of Nile discharge and sapropel S1 formation in the early to middle Holocene eastern Mediterranean. Paleoceanography 29, 343-356. http://dx.doi.org/10.1002/ 2013PA002553.

Hennekam, R., Donders, T.H., Zwiep, K., de Lange, G.J., 2015. Integral view of Holocene precipitation and vegetation changes in the Nile catchment area as inferred from its delta sediments. Quat. Sci. Rev. 130, 189-199.

Homoky, W.B., Severmann, S., Mills, R.A., Statham, P.J., Fones, G.R., 2009. Pore-fluid Fe isotopes reflect the extent of benthic Fe redox cycling: evidence from continental shelf and deep-sea sediments. Geology 37, 751-754.

Johnson, C.M., Roden, E.E., Beard, B.L., Welch, S.A., 2005. Experimental constraints on Fe isotope fractionation during magnetite and Fe carbonate formation coupled to dissimilatory hydrous ferric oxide reduction. Geochim. Cosmochim. Acta 69, 963-993.

Johnson, C.M., Beard, B.L., Roden, E.E., 2008. The iron isotope fingerprints of redox and biogeochemical cycling in modern and ancient earth. Annu. Rev. Earth Planet. Sci. 36, 457-493.

Kallel, N., Duplessy, J.C., Labeyrie, L., Fontugne, M., Paterne, M., Montacer, M., 2000. Mediterranean pluvial periods and sapropel formation during the last 200,000 years. Palaeogeogr. Palaeoclimatol. Palaeoecol. 157, 45-58.

Kendall, B., Dahl, T.W., Anbar, A.D., 2017. Good Golly, Why Moly? The stable isotope geochemistry of molybdenum. In: Teng, F.-Z., Watkins, J.M., Dauphas, N. (Eds.) Non-Traditional Stable Isotopes. Rev. Mineral. Geochem. 82. pp. 683-732.

Kerl, C.F., Lohmayer, R., Bura-Nakic, E., Vance, D., Planer-Friedrich, B., 2017. Experimental confirmation of isotope fractionation in thiomolybdates using ion chromatographic separation and detection by Multicollector ICPMS. Anal. Chem. 89, 3123-3129.

Krom, M.D., Cliff, R.A., Eijsink, L.M., Herut, B., Chester, R., 1999. The characterization of Saharan dusts and Nile particulate matter in surface sediments from the Levantine basin using Sr isotopes. Mar. Geol. 155, 319-325.

Kudnt, T., Schmiedl, G., Ehrmann, W., Hamann, Y., Andersen, N., 2008. Stable isotope composition of Holocene benthic foraminifers from Eastern Mediterannean Sea: past changes in productivity and deep water oxygenation. Palegeogr. Paleoclimatol. Paleecol. 268, 106-115.

de Lange, G.J., Thomson, J., Reitz, A., Slomp, C.P., Speranza Principato, M., Erba, E., Corselli, C., 2008. Synchronous basin wide formation and redox-controlled preservation of a Mediterranean sapropel. Nat. Geosci. 1, 606-610.

Langgut, D., Almogi-Labin, A., Bar-Matthews, M., Weinstein-Evron, M., 2011. Vegetation and climate changes in the South Eastern Mediterranean during the Last GlacialInterglacial cycle (86 ka): new marine pollen record. Quat. Sci. Rev. 30, 3960-3972.

Larrasoana, J.C., Roberts, A.P., Rohling, E.J., Winklhofer, M., Wehausen, R., 2003. Three million years of monsoon variability over the northern Sahara. Clim. Dyn. 21, 689-698.

Lyons, T.W., Severmann, S., 2006. A critical look at paleoredox proxies: new insights from modern euxinic marine basins. Geochim. Cosmochim. Acta 70, 5698-5722.

Lyons, T.W., Anbar, A.D., Severmann, S., Scott, C., Gill, B.C., 2009. Tracking euxinia in the ancient ocean: a multiproxy perspective and Proterozoic case study. Annu. Rev. Earth Planet. Sci. 37, 507-534.

deMenocal, P., Ortiz, J., Guilderson, T., Adkins, J., Sarnthein, M., Baker, L., Yarusinsky, M., 2000. Abrupt onset and termination of the African Humid Period: rapid climate responses to gradual insolation forcing. Quat. Sci. Rev. 19, 347-361.

Murat, A., Got, H., 1987. Middle and Late Quaternary depositional sequences and cycles in the eastern Mediterranean. Sedimentology 34, 885-899.

Nägler, T.F., Neubert, N., Böttcher, M.E., Dellwig, O., Schnetger, B., 2011. Molybdenum isotope fractionation in pelagic euxinia: evidence from the modern Black and Baltic
Seas. Chem. Geol. 289, 1-11.

Nägler, T.F., Anbar, A.D., Archer, C., Goldberg, T., Gordon, G.W., Greber, N.D., Siebert, C., Sohrin, Y., Vance, D., 2014. Proposal for an international molybdenum isotope measurement standard and data representation. Geostand. Geoanal. Res. 38, $149-151$.

Neubert, N., Nägler, Th.F., Böttcher, M.E., 2008. Sulfidity controls molybdenum isotope fractionation in euxinic sediments: evidence from the modern Black Sea. Geology 36, 775-778.

Osborne, A.H., Vance, D., Rohling, E.J., Barton, N., Rogerson, M., Fello, N., 2008. A humid corridor across the Sahara for the migration of early modern humans out of Africa, 120,000 years ago. Proc. Natl. Acad. Sci. U. S. A. 105 (16), 444-16,447.

Passier, H.F., Middelburg, J.J., van Os, B.J.H., de Lange, G.J., 1996. Diagenetic pyritization under eastern Mediterranean sapropels caused by downward sulphide diffusion. Geochim. Cosmochim. Acta 60, 751-763.

Passier, H.F., de Lange, G.J., Middelburg, J.J., Böttcher, M.E., 1997. Pyrite contents, microtextures, and sulphur isotopes in relation to formation of the youngest eastern Mediterranean sapropel. Geology 25, 519-522.

Passier, H.F., Middelburg, J.J., de Lange, G.J., Böttcher, M.E., 1999. Modes of sapropel formation in the eastern Mediterranean: some constraints based on pyrite properties. Mar. Geol. 153, 199-219.

Poitrasson, F., Vieira, L.C., Seyler, P., dos Santos Pinheiro, G.M., Mulholland, D.S., Bonnet, M.-P., Martinez, J.-M., Lima, B.A., Boaventura, G.R., Chmeleff, J., 2014. Iron isotope composition of the bulk waters and sediments from the Amazon River Basin. Chem. Geol. 377, 1-11.

Poulson, R.L., Siebert, C., McManus, J., Berelson, W.M., 2006. Authigenic molybdenum isotope signatures in marine sediments. Geology 34, 617-620.

Poulson-Brucker, R.L., McManus, J., Severmann, S., Berelson, W.M., 2009. Molybdenum behavior during early diagenesis: insights from Mo isotopes. Geochem. Geophys. Geosyst. 10. http://dx.doi.org/10.1029/2008GC002180.

Poulson-Brucker, R.L., McManus, J., Poulton, S.W., 2012. Molybdenum isotope fractionation observed under anoxic experimental conditions. Geochem. J. 46, 201-209.

Poulton, S.W., 2003. Sulfide oxidation and iron dissolution kinetics during the reaction of dissolved sulfide with ferrihydrite. Chem. Geol. 202, 79-94.

Poulton, S.W., Canfield, D.E., 2005. Development of a sequential extraction procedure for iron: implications for iron partitioning in continentally derived particulates. Chem. Geol. 214, 209-221.

Poulton, S.W., Canfield, D.E., 2011. Ferruginous conditions: a dominant feature of the ocean through earth's history. Elements 7 (2), 107-112.

Poulton, S.W., Raiswell, R., 2002. The low-temperature geochemical cycle of iron: from continental fluxes to marine sediment deposition. Am. J. Sci. 302, 774-805.

Poulton, S.W., Krom, M.D., Raiswell, R., 2004. A revised scheme for the reactivity of iron (oxyhydr)oxide minerals towards dissolved sulfide. Geochim. Cosmochim. Acta 68, 3703-3715.

Poulton, S.W., Fralick, P.W., Canfield, D.E., 2010. Spatial variability in oceanic redox structure 1.8 billion years ago. Nat. Geosci. 3, 486-490.

Raiswell, R., Canfield, D.E., 1998. Sources of iron for pyrite formation in marine sediments. Am. J. Sci. 298, 219-245.

Raiswell, R., Canfield, D.E., 2012. The iron biogeochemical cycle past and present. Geochem. Perspect. 1 (220 pp.).

Reitz, A., Wille, M., Nägler, T.F., de Lange, G.J., 2007. Atypical Mo isotope signatures in eastern Mediterranean sediments. Chem. Geol. 245, 1-8.

Revel, M., Ducassou, E., Grousset, F.E., Bernasconi, S.M., Migeon, S., Revillon, S., Mascle, J., Murat, A., Zaragosi, S., Bosch, D., 2010. 100,000 years of Africanmonsoon variability recorded in sediments of the Nile margin. Quat. Sci. Rev. 29, 1342-1362.

Revel, M., Colin, C., Bernasconi, S., Combourieu-Nebout, N., Ducassou, E., Grousset, F., Rolland, Y., Migeon, S., Bosch, D., Brunet, P., Zhao, Y., Mascle, J., 2014. 21,000 years of Ethiopian African monsoon variability recorded in sediments of the western Nile deep-sea fan. Reg. Environ. Chang. 14, 1685-1696. http://dx.doi.org/10.1007/ s10113-014-0588-x.

Rohling, E.J., 1994. Review and new aspects concerning the formation of the eastern Mediterranean sapropels. Mar. Geol. 122, 1-28.

Rohling, E.J., Marino, G., Grant, K.M., 2015. Mediterranean climate and oceanography, and the periodic development of anoxic events (sapropels) (2015). Earth-Sci. Rev. 143, 62-97.

Rossignol-Strick, M., 1985. Mediterranean Quaternary sapropels: an immediate response to of the African Monsoon to variation of insolation. Paleogeogr. Paleoclimatol. Paleoecol. 49, 237-265.

Rossignol-Strick, M., Nesteroff, V., Olive, P., Vergnaud-Grazzini, C., 1982. After the deluge: Mediterranean stagnation and sapropel formation. Nature 295, 105-110.

Scheiderich, K., Zerkl, A.L., Helz, G.R., Farquhar, J., Walker, R.J., 2010. Molybdenum isotope, multiple sulfur isotope, and redox-sensitive element behavior in early Pleistocene Mediterranean sapropels. Chem. Geol. 279, 134-144.

Schmiedl, G., Kuhnt, T., Ehrmann, W., Emeis, K.-C., Hamann, Y., Kotthoff, U., 2010. Climatic forcing of the eastern Mediterranean Sea deep-water formnation and benthic ecosystems during the past 22,000 years. Quat. Sci. Rev. 29, 3006-3020.

Scholz, F., Severmann, S., McManus, J., Hensen, C., 2014. Beyond the Black Sea paradigm. The sedimentary fingerprint of an open-marine iron shuttle. Geochim. Cosmochim. Acta 127, 368-380.

Scrivner, A.E., Vance, D., Rohling, E.J., 2004. New neodymium isotope data quantify Nile involvement in Mediterranean anoxic episodes. Geology 32, 565-568.

Severmann, S., Johnson, C.M., Beard, B.L., McManus, J., 2006. The effect of early diagenesis on the Fe isotope compositions of pore waters and authigenicminerals in continental margin sediments. Geochim. Cosmochim. Acta 70, 2006-2022.

Severmann, S., Lyons, T.W., Anbar, A., McManus, J., Gordon, G., 2008. Modern ironisotope perspective on Fe shuttling in the Archean and the redox evolution ofancient oceans. Geology 36, 487-490. 
Severmann, S., McManus, J., Berelson, W.M., Hammond, D.E., 2010. The continental shelf benthic iron flux and its isotope composition. Geochim. Cosmochim. Acta 74 3984-4004.

Siebert, C., Nägler, T.F., Kramers, J.D., 2001. Determination of molybdenum isotope fractionation by double-spike multicollector inductively coupled plasma mass spec trometry. Geochem. Geophys. Geosyst. 2 (2000GC00124).

Siebert, C., Nägler, T.F., von Blankenburg, F., Kramers, J.D., 2003. Molybdenum isotope records as a proxy for paleoceanography. Earth Planet. Sci. Lett. 211, 159-171.

Siebert, C., McManus, J., Bice, Angela, Poulson, R., Berelson, W.P., 2006. Molybdenum isotope signatures in continental margin sediments. Earth Planet. Sci. Lett. 241, 723-733.

Staubwasser, M., von Blanckenburg, F., Schönberg, K., 2006. Iron isotopes in the early marine diagenetic iron cycle. Geology 34, 629-632.

Staubwasser, M., Schoenberg, R., von Blanckenburg, F, Krïger, S., Pohl, C., 2013. Isotope fractionation between dissolved and suspended particulate Fe in the oxic and anoxic water column of the Baltic Sea. Biogeosciences 10, 233-245.

Stookey, L.L., 1970. Ferrozine - a new spectrophotometric reagent for iron. Anal. Chem. 42, 779-781.

Tachikawa, K., Vidal, L., Cornuault, M., Garcia, M., Pothin, A., Sonzogni, C., Bard, E., Menot, G., Revel, M., 2015. Eastern Mediterranean Sea circulation inferred from the conditions of S1 sapropel deposition. Clim. Past 11, 855-886.

Tahata, M., Sawaki, Y., Yoshiya, K., Nishizawa, M., Komiya, T., Hirata, T., Yoshida, N., Maruyama, S., Windley, B.F., 2015. The marine environments accompanying the Neoproterozoic glaciations: evidence from C, $\mathrm{Sr}$ and Fe isotope ratios in the Hecla Hock Supergroup in Svalbard. Precambrian Res. 263, 19-42.

Teutsch, N., Schmid, M., Mueller, B., Halliday, A.N., Burgmann, H., Wehrli, B., 2009 Large iron isotope fractionation at the oxic-anoxic boundary in Lake Nyos. Earth Planet. Sci. Lett. 285, 52-60.

Thomson, J., Mercone, D., de Lange, G.J., van Santvoort, P.J.M., 1999. Review of recent advances in the interpretation of Eastern Mediterranean sapropel S1 from geochemical evidence. Mar. Geol. 153, 77-89.

Tossell, J.A., 2005. Calculating the partitioning of the isotopes of Mo between oxidic and sulfidic species in aqueous solution. Geochim. Cosmochim. Acta 69, 2981-2993.

Tribovillard, N., Algeo, T.J., Lyons, T., Riboulleou, T., 2006. Trace elements as paleoredox and paleoproductivity proxies: an update. Chem. Geol. 232, 12-32.

Vance, D., Scrivner, A.E., Beney, P., Staubwasser, M., Henderson, G.F., Stowell, N.C., 2004. The use of foraminifera as a record of past neodymium isotope composition of seawater. Paleoceanography 19http://dx.doi.org/10.1029/2003PA000957. (PA2009).

van Helmond, N.A.G.M., Hennekam, R., Donders, T.H., Bunnik, F.P.M., De Lange, G.J., Brinkhuis, H., Sangiorgi, F., 2015. Marine productivity leads organic matter preservation in sapropel S1: palynological evidence from a core east of the Nile River outflow. Quat. Sci. Rev. 108, 130-138.

Van Santvoort, P.J.M., De Lange, G.J., Thomson, J., Cussen, H., Wilson, T.R.S., Krom, M.D., Strohle, K., 1996. Active post-depositional oxidation of the most recent sapropel (S1) in the eastern Mediterranean. Geochim. Cosmochim. Acta 60 4007-4024.

Wagner, M., Chappaz, A., Lyons, T.W., 2017. Molybdenum speciation and burial path ways in weakly sulfidic environments: insights from XAFS. Geochim. Cosmochim. Acta 206, 18-29.

Warning, B., Brumsack, H.-J., 2000. Trace metal signatures of eastern Mediterranean sapropels. Paleogeogr. Paleoclimatol. Paleoecol. 158, 293-309.

Wasylenki, L.E., Rolfe, B.A., Weeks, C.L., Spiro, T.G., Anbar, A.D., 2008. Experimental investigation of the effects of temperature and ionic strength on Mo isotope fractionation during adsorption to manganese oxides. Geochim. Cosmochim. Acta 72, 5997-6005.

Zegeye, A., Bonneville, S., Benning, L., Sturm, A., Fowle, D.A., Jones, C., Canfield, D.E. Ruby, C., Maclean, L.C., Nomosatryo, S., Crowe, S.A., Poulton, S.W., 2012. Green rust formation controls nutrient availability in a ferruginous water column. Geology 40, 599-602. 